\title{
Dispersive nonlinear geometric optics
}

\author{
Phillipe Donnat \\ Commisariat de l'Énergie Atomique, Centre d'Étude de Limeil Valenton, \\ 94195 Villeneuve St. Georges, France \\ Jeffrey Rauch \\ Department of Mathematics, University of Michigan, Ann Arbor, Michigan 48109
}

(Received 22 April 1996; accepted for publication 29 July 1996)

We construct infinitely accurate approximate solutions to systems of hyperbolic partial differential equations which model short wavelength dispersive nonlinear phenomena. The principal themes are the following. (1) The natural framework for the study of dispersion is wavelength $\epsilon$ solutions of systems of partial differential operators in $\epsilon \partial$. The natural $\epsilon$-characteristic equation and $\epsilon$-eikonal equations are not homogeneous. This corresponds exactly to the fact that the speeds of propagation, which are called group velocities, depend on the length of the wave number. (2) The basic dynamic equations are expressed in terms of the operator $\epsilon \partial_{t}$. As a result growth or decay tends to occur at the catastrophic rate $e^{c t / \epsilon}$. The analysis is limited to conservative or nearly conservative models. (3) If a phase $\phi(x) / \epsilon$ satisfies the natural $\epsilon$-eikonal equation, the natural harmonic phases, $n \phi(x) / \epsilon$, generally do not. One needs to impose a coherence hypothesis for the harmonics. (4) In typical examples the set of harmonics which are eikonal is finite. The fact that high harmonics are not eikonal suppresses the wave steepening which is characteristic of quasilinear wave equations. It also explains why a variety of monochromatic models are appropriate in nonlinear settings where harmonics would normally be expected to appear. (5) We study wavelength $\epsilon$ solutions of nonlinear equations in $\epsilon \partial$ for times $O(1)$. For a given system, there is a critical exponent $p$ so that for amplitudes $O\left(\epsilon^{p}\right)$, one has simultaneously smooth existence for $t=O(1)$, and nonlinear behavior in the principal term of the approximate solutions. This is the amplitude for which the time scale of nonlinear interaction is $O(1)$. (6) The approximate solutions have residual each of whose derivatives is $O\left(\epsilon^{n}\right)$ for all $n>0$. In addition, we prove that there are exact solutions of the partial differential equations, that is with zero residual, so that the difference between the exact solution and the approximate solutions is infinitely small. This is a stability result for the approximate solutions. (c) 1997 American Institute of Physics.

[S0022-2488(97)00602-6]

\section{INTRODUCTION}

This paper presents a method for constructing rigorously justified infinitely accurate approximate solutions to systems of hyperbolic partial differential equations which model short wavelength dispersive nonlinear phenomena. A tool of general utility is created. It is important to note that there are a variety of more or less ad hoc methods to arrive at the leading term of the approximate solutions. We provide a framework which justifies many such arguments and can serve to arbitrate controversies where contradictory simplifications are proposed.

The classical use of the expression dispersion is to describe the fact that white light is split into a rainbow of colors on passing through a prism. The shorter wavelength light, bluish in color, is bent more than the longer wavelengths which are redish in color. The reason is that the short wavelength light travels more slowly through glass than does the longer wavelengths. This dependence of speed on wavelength is called dispersion.

The cause of this phenomenon is that the light forces the electrons in atoms and molecules to 
oscillate. The oscillating electrons emit light. The total field is a combination of the incident and emitted fields which in nonobvious fashion leads to an effective speed of propagation which is different than the speed in a vacuum. The resonant frequency of the atomic oscillators in glass is in the near ultraviolet so that blue tones are closer to resonance than red. This explains why dispersion is stronger for shorter wavelengths. The reason that glass is dispersive and air is not is because there are many more atoms per unit volume in glass. The reader is referred to Ref. 1 for a particularly good presentation of the physics. The key is that the frequency of the exciting light and the resonant frequencies of the atoms are both very large and of comparable magnitude. In the infrared and x-ray regions of the electromagnetic spectrum, dispersive effects are much less important. The need for this tuning is well expressed in the introduction to dispersion in Ref. 2:

“...to study the important subject of rapidly varying electromagnetic fields whose frequencies are not restricted to be small in comparison with the frequencies which characterize the establishment of the electric and magnetic polarisation of the substances concerned."

In units so that the speed of light in vacuum is equal to one, wavelength $\epsilon$ electromagnetic waves have period $O(\epsilon / 2 \pi)$. The atomic oscillators to be near resonance will also have period of the same order. A harmonic oscillator with this frequency has an equation of the form

$$
\epsilon^{2} \frac{d^{2} p}{d t^{2}}+p=0
$$

Note in particular the appearance of the differential operator $\epsilon d / d t$. Pursued systematically as in Ref. 3, this idea leads to models for linear and nonlinear dispersion, as in Sec. II, which have the following form. An unknown $\mathbf{R}^{N}$ - or $\mathbf{C}^{N}$ - valued field $u(t, y)$ defined for $x:=(t, y) \in \mathbf{R}^{1+d}$ satisfies a system of partial differential equations

$$
L\left(u, \epsilon \partial_{x}\right) u+F(u)=0,
$$

where

$$
L(u, \xi):=\sum_{\mu=0}^{d} A_{\mu}(u) \xi_{\mu}+L_{0}:=L_{1}(u, \xi)+L_{0} .
$$

Order $J$ hypothesis: The nonlinear functions $F$ and $A_{\mu}$ are smooth on a neighborhood of 0 , and the nonlinear terms are of order $J \geqslant 2$ in the sense that

$$
|\alpha| \leqslant J-2 \Rightarrow \partial_{u, \bar{u}}^{\alpha} A_{\mu}(0)=0 \quad \text { and } \quad|\beta| \leqslant J-1 \Rightarrow \partial_{u, \bar{u}}^{\beta} F(0)=0 .
$$

The system is symmetric hyperbolic in the sense that

$$
A_{\mu}(u)=A_{\mu}(u)^{*} \quad \text { and } \quad A_{0}(0)>0 .
$$

The simplest such equation is $\epsilon \partial_{t} u= \pm c u$ whose solutions are of the form $e^{ \pm c t / \epsilon} f(y)$. Unless $c$ is purely imaginary the solutions are either negligibly small or explosively large. In order that our system be neither strongly dissipative nor strongly explosive, we assume that

$$
L_{0}=-L_{0}^{*}
$$

The linearized equation at $u=0$ is

$$
L\left(0, \epsilon \partial_{x}\right) v=0
$$


This is a constant coefficient system of linear $\epsilon \partial$ differential equations. The hypothesis (1.5) is equivalent to conservation of the quantity $\left\langle A_{0} u, u\right\rangle$ for this linearized system. The discussion of dispersion suggests that one seek solutions of wavelength $O(\epsilon)$ and with that in mind, note that there exist plane wave solutions $v=e^{i \beta x / \epsilon} \mathbf{r}$ exactly when $\mathbf{r} \in \operatorname{ker} L(0, i \beta)$. This kernel is nontrivial exactly when $\beta$ is a solution of the $\epsilon$-characteristic equation

$$
0=\operatorname{det} L(0, \beta)=\operatorname{det}\left(\sum_{\mu=0}^{d} i \beta_{\mu} A_{\mu}(0)+L_{0}\right) .
$$

The solutions $\beta$ are by definition the points of the $\epsilon$-characteristic variety, denoted $\epsilon$ char. The presence of the term $L_{0}$ in (1.7) shows that this variety depends on the lower-order terms in the equation, and is defined by an equation which need not be homogeneous in $\beta$.

Definition: For any $\beta \in \mathbf{R}^{1+d}$, let $\pi(\beta)$ denote orthogonal projection onto the kernel of $L(0, i \beta)$.

In particular $\pi(\beta)$ is nonzero exactly when $\beta \in \epsilon$ char.

There are at least three distinct ways to arrive at the ansatz for the approximate solutions of this article. The idea of modulated plane waves, sometimes called the slowly varying envelope hypothesis, is the most classical. A second is Whitham's averaged Lagrangian method ${ }^{4}$ which requires a variational form. Both of these methods are intuitively appealing and predict the leading term in an approximate solution. Since the leading term is the most interesting, one might think that such methods should be sufficient. However, if the approximate solution is constructed only up to the principal term, then the residual in the equation is of the same order of magnitude as the approximation itself. This explains in part why it is difficult to show that such approximations are in fact accurate. One of the key recent advances for nondispersive problems, by Choquet-Bruhat, Majda, Rosales, Hunter, Keller, Joly, Metivier, and Rauch, ${ }^{5-13}$ is the development of a third strategy which follows the lines of WKB expansions is a systematic way. In addition to flexibility, this approach has the dual advantage of often suggesting improved approximations, and wide applicability. This is partly why it is the only one of the approaches which has lead to rigorous results for nonlinear problems. A survey of recent progress including a more complete bibliography can be found in Ref. 14 .

The natural starting point for all approaches are problems which are explicitly solvable. Consider the linear constant coefficient oscillatory initial value problem

$$
L\left(0, \epsilon \partial_{x}\right) v^{\epsilon}=0, \quad v^{\epsilon}(0, y)=g(y) e^{i \psi(y) / \epsilon} .
$$

The solution is given exactly by

$$
v^{\epsilon}(t, y)=\frac{1}{(2 \pi)^{d}} A_{0}^{-1 / 2} \int_{\mathbf{R}^{d}} \int_{\mathbf{R}^{d}} e^{i H(\epsilon, \eta) t} e^{i(x-y) \cdot \eta} A_{0}^{1 / 2} g(y) e^{i \psi(y) / \epsilon} d y d \eta,
$$

where

$$
H(\epsilon, \eta):=A_{0}^{-1 / 2}\left(\frac{i L_{0}}{\epsilon}-\sum_{j=0}^{d} \eta_{j} A_{j}\right) A_{0}^{-1 / 2} .
$$

If the $\epsilon$-characteristic variety has the property that over the points $d \psi(y)$ it consists of a finite number of smooth nonintersecting sheets in the sense that for $\eta$ in a neighborhood of

$$
\{d \psi(y): y \in \operatorname{supp} g\}
$$

the equation 


$$
L(0, i \tau, i \eta)=0
$$

has exactly $K$ distinct roots

$$
\tau_{1}(\eta)<\tau_{2}(\eta)<\cdots<\tau_{K}(\eta),
$$

then there are $K$ natural phases for the problem (1.8) namely, the solutions of the eikonal equation

$$
\operatorname{det} L(0, i d \phi)=0, \quad \phi(0, y)=\psi(y),
$$

which are the solutions of the $K$ reduced equations

$$
\partial_{t} \phi_{k}=\tau_{k}\left(\partial_{y} \phi_{k}\right), \quad \phi_{k}(0, y)=\psi(y), \quad 1 \leqslant k \leqslant K .
$$

The nonlinear equations (1.12) are uniquely solvable for small time. Applying the method of stationary phase for such times shows that as $\epsilon \rightarrow 0$ the solution given by (1.9) is equal to a sum of $K$ terms

$$
v^{\epsilon}=\sum_{k=1}^{K} v_{k}^{\epsilon}, \quad v_{k}^{\epsilon}=a_{k}(\epsilon, x) e^{l \phi_{k}(x) / \epsilon}, \quad a_{k}(\epsilon, x) \sim \sum_{j=0}^{\infty} \epsilon^{j} a_{k, j}(x) .
$$

The leading profiles $a_{k, 0}$ are polarized and have initial values according to

$$
\pi\left(d \phi_{k}\right) a_{k, 0}=a_{k, 0}, \quad a_{k, 0}(0, y)=\pi\left(d \phi_{k}(x)\right) g(y) .
$$

They are determined by a set of ordinary differential equations, aka transport equations, along curves in space-time, aka, rays, moving at the natural group velocities $-\partial_{\eta} \tau_{k}(d \phi(x))$. In Proposition 3.3 we will see that these transport equations are equivalent to the system

$$
\pi(d \phi(x)) L_{1}\left(0, \partial_{x}\right) \pi(d \phi(x)) a_{k, 0}(x)=0 .
$$

In addition to the asymptotic evaluation of exact solutions we would also like to cite the paper by Lewis, ${ }^{15}$ who constructs asymptotic solutions of dispersive linear problems which need not have explicit solutions. His models for the dispersion of light do not have natural energy estimates which prevents him from proving that his approximate solutions are close to exact solutions. We give an example where approximate solutions are in fact far from exact solutions after Theorem 3.7.

There is a large literature on relaxation problems which is also related to our work. The problems are singular limits of nonlinear $\epsilon \partial$ equations for which decay like $e^{-t / \epsilon}$ is present in some modes. The goal is to extract a correct description for a relaxed system in which these modes are not present (see Ref. 16 and its bibliography). An important part of the analysis are hypotheses which exclude explosive modes and guarantee stability as does our conservation hypothesis (1.5).

It is reasonable to seek approximate solutions similar to those in (13) but in contexts where they are not derived by an asymptotic analysis of an explicit solution, in particular for nonlinear problems. With this in mind suppose that $\phi(x)$ satisfies the eikonal equation. The first nonlinear phenomenon to discuss is the creation of harmonics. Nonlinear functions applied to expressions of the form $a(\epsilon, x) e^{i \phi(x) / \epsilon}$ will produce harmonics, that is expression with phases $n \phi$ for $n \in \mathbf{Z}$. Negative values of $n$ come from nonlinear functions such as the complex conjugate. The value $n=0$ appears clearly for example for the nonlinear function $|u|^{2}$. An important difference between the case of dispersive geometric optics and the nondispersive case (for example, Refs. 9-13) is that if $\phi$ satisfies the eikonal equation, then for most $n, n \phi$ does not satisfy the eikonal equation. The simplest case is the Klein-Gordon equation $\epsilon^{2} \square u+u=0$. If $\phi$ satisfies the eikonal equation 
$\phi_{t}^{2}=1+\left|\phi_{y}\right|^{2}$, then for $n \neq \pm 1, n \phi$ satisfies the eikonal equation at no points. For $n=-1, n \phi$ satisfies the eikonal equation at all points. This is an example for which the following hypothesis is satisfied.

Coherence hypothesis: The phase $\phi$ is a smooth real solution of the eikonal equation on $\Omega$ and or each $m \in \mathbf{Z} \backslash \mathbf{0}$. If $L(\operatorname{imd} \phi(x))$ is singular for one $x \in \Omega$, then it is singular for all $x \in \Omega$. When $L(i m d \phi(x))$ is singular, the matrix $\pi(m d \phi(x))$ is assumed to be a smooth function of $x$.

Analogous coherence hypotheses were introduced by Majda and Rosales ${ }^{6}$ in the study of multiphase nondispersive problems. The interaction of harmonics of dispersive systems is in this sense analogous to the interaction of distinct phases for nondispersive systems.

The next hypothesis avoids some small divisor problems as $|n| \rightarrow \infty$ and is satisfied in all the physical examples we have studied.

Strong finiteness hypothesis: $L_{1}(i d \phi(x))$ is nonsingular for all $x \in \Omega$.

This hypothesis implies that

$$
\mathscr{C l}:=\{m \in \mathbf{Z}: \operatorname{det} L(\operatorname{imd} \phi)=0\}
$$

is finite. The principal term in our approximate solutions is a sum of terms of the form (1.13), one term for each of the eikonal phases $m \phi$,

$$
u^{\epsilon} \approx \epsilon^{p} a_{0}(x, \phi(x) / \epsilon), \quad a_{0}(x, \theta):=\sum_{m \in \mathscr{C}} a_{m}(x) e^{i \theta}, \quad \pi(m d \phi(x)) a_{m}(x)=a_{m}(x) .
$$

Roughly $u^{\epsilon}$ is a finite family of nonlinearly interacting dispersive waves whose amplitude, $\epsilon^{p}$ is discussed in the next paragraph.

The second nonlinear effect to take care of is that the strength of interaction and therefore the time scale for interaction depends on the amplitude of the wave. The amplitude of the solution (1.17) is $\epsilon^{p}$. The exponent $p$ is chosen so that the time scale for the nonlinear interactions is $O(1)$. This vague phrase means that one cannot ignore the nonlinear effects if one wants an approximation for times independent of $\epsilon$, but for times tending to zero with $\epsilon$ they can be ignored. We present two independent computations of $p$. The second is at the beginning of Sec. IV A. For the first, suppose that $u$ and $\epsilon \partial u^{\epsilon}$ are $O\left(\epsilon^{p}\right)$. Then (1.1) takes the form

$$
\left(L_{1}\left(0, \partial_{x}\right)+\frac{1}{\epsilon} L_{0}\right) u^{\epsilon}=\frac{1}{\epsilon} O\left(\epsilon^{p J}\right),
$$

where the right-hand side comes from the nonlinear terms satisfying (1.3). Thanks to the conservation hypothesis, the propagators for the linear operator on the left are uniformly bounded on the standard Sobolev spaces. Thus the effect of the nonlinear terms over times of order one is estimated to be of order $\epsilon^{p-1}$. The critical exponent $p$ is chosen so that this is equal to $\epsilon^{p}$, the amplitude of the solutions studied. Thus

$$
p=\frac{1}{J-1}
$$

In Sec. V interaction coefficients $c_{m}\left(x,\left\{a_{\mu}(x)\right\}\right)$ are defined depending on the phase $\phi$ and the derivatives of $L(u,$.$) and F(u)$ with respect to $u$ at $u=0$. Each function $c_{m}$ is a homogeneous polynomial in $\left\{a_{\mu}(x)\right\}$ of degree $J$. The principal profile $a_{0}$ in (1.17) is uniquely determined from its initial data by the coupled semilinear symmetric hyperbolic systems

$$
\pi(m d \phi(x)) L_{1}\left(0, \partial_{x}\right) \pi(m d \phi(x)) a_{m}+c_{m}\left(x,\left\{a_{\mu}(x)\right\}\right)=0 .
$$


The operators on the left are those in (15). Proposition 3.3 shows that where the characteristic variety is simple they are transport operators at the group velocity. To guarantee uniqueness one needs to suppose that the domain $\Omega$ on which one works satisfies the following condition.

Determinacy hypothesis: $\Omega \cap\left(\left[0, T_{1}\right] \times \mathbf{R}^{d}\right)$ is a domain of determinacy for each of the symmetric hyperbolic operators $\pi(m d \phi) L_{1}\left(\partial_{x}\right) \pi(m d \phi), m \in \mathscr{l l}$.

Proposition 3.2 shows that this is automatically satisfied if $\Omega \cap\left(\left[0, T_{1}\right] \times \mathbf{R}^{d}\right)$ is a domain of determinacy for $L_{1}\left(0, \partial_{x}\right)$

The principal term can be corrected to give infinitely accurate approximations. These are the dispersive analogue of the constructions of Joly-Rauch and Gues in Refs. 9, 17, and 18. The proofs of all these authors are descendents of the seminal article of $\mathrm{Lax}^{19}$ showing that the geometric optics approximate in the linear case is easily justified by constructing an infinitely accurate approximation solution and then using energy estimates.

The nonlinear dispersive analogue of Lax's result is harder. For one, the solutions tend to infinity in the norms in which the Cauchy problem is well set. This suggests that they may become increasingly sensitive to perturbations in the data, and therefore the approximate solutions need not be accurate. In fact they are accurate as we prove in Sec. VI.

The approximate solutions have the form

$$
u^{\epsilon}(x)=\epsilon^{p} a(\epsilon, x, \phi(x) / \epsilon), \quad \text { with } a(\epsilon, x, \theta) \sim \epsilon^{p} \sum_{j \in p \mathbf{N}} \epsilon^{j} a_{j}(x, \theta) .
$$

The functions $a(\epsilon, x, \theta)$ and $a_{j}(x, \theta)$ are smooth in all their arguments and periodic in $\theta$. To describe the main results introduce the projector $\Pi$ acting on trigonometric polynomials $d(x, \theta)=\sum d_{n}(x) e^{i n \theta}$ by

$$
\boldsymbol{\Pi} d:=\sum_{n \in \mathbf{N}} \pi(n d \phi(x)) d_{n}(x) e^{i n \theta} .
$$

The next theorem shows that infinitely accurate approximate solutions of the form (1.20) are uniquely determined once initial data are given for $\Pi a_{j}(0, y)$.

Theorem: Suppose that $T_{1}>0$ and for $j \geqslant 0$

$$
g_{j}(y, \theta) \in C_{0}^{\infty}\left(\mathbf{R}^{d} \times S^{1}\right), \quad \text { supp } g \subset(\Omega \cap\{t=0\}) \times S^{1} \quad \text { and } \Pi g_{j}=g_{j} .
$$

Then there is a $\left.T \in] 0, T_{1}\right]$ and a unique solution $a_{0} \in C_{(0)}^{\infty}\left((\Omega \cap\{0 \leqslant t \leqslant T\}) \times S^{1}\right)$ of $(1.19)$ such that $\Pi a_{0}(0, y)=g_{0}(y)$. With this $T$, there are unique functions $a_{j} \in C_{(0)}^{\infty}\left((\Omega \cap\{0 \leqslant t \leqslant T\}) \times S^{1}\right)$ satisfying the initial conditions $\Pi a_{j}(0, y)=g_{j}(y)$ and so that if $u^{\epsilon}$ satisfies (1.20), then for all $\alpha$ and $M$ one has

$$
\partial^{\alpha}\left(L\left(u^{\epsilon}, \epsilon \partial_{x}\right) u^{\epsilon}+F\left(u^{\epsilon}\right)\right)=O\left(\epsilon^{M}\right)
$$

uniformly on $\Omega \cap\{0 \leqslant t \leqslant T\}$.

This result is proved in Sec. IV in the semilinear case and in Sec. V for the quasilinear case. That the approximate solution is infinitely close to exact solutions is given by the following result which is weaker than that proved in Sec. VI.

Theorem: Suppose that $u^{\epsilon}$ and $T$ are as in the previous theorem and that $v^{\epsilon}$ is the unique local solution of the initial value problem

$$
L\left(v^{\epsilon}, \partial\right) v^{\epsilon}+F\left(v^{\epsilon}\right)=0, \quad v^{\epsilon}(0, y)=u^{\epsilon}(0, y) .
$$

Then for small positive $\epsilon, v^{\epsilon}$ exists and is smooth on $[0, T] \times \mathbf{R}^{d}$ and for all $\alpha$ and $M$ one has

$$
\partial_{x}^{\alpha}\left(u^{\epsilon}-v^{\epsilon}\right)=O\left(\epsilon^{M}\right)
$$


uniformly on $[0, T] \times \mathbf{R}^{d}$.

In Sec. II we present three models from nonlinear optics, one linear, one semilinear, and one quasilinear which together with the Klein-Gordon equation serve as examples throughout the exposition. In Sec. III, linear dispersion is discussed. Here many fundamental relations needed in the sequel are proved and the scheme for handling the recursive definition of the profiles $a_{j}$ is developed. While much of this material is known, we think that much is also new. In this section we also show that for the simple nonconservative example $L(\epsilon \partial):=\epsilon^{2} \square-1$ there are asymptotic solutions with infinitely small residual, $L(\epsilon \partial) u \sim 0$, so that $u^{\epsilon}$ and $v^{\epsilon}$ have disjoint support for times $t \sim 1$. In Sec. IV we construct approximate solutions with infinitely small residual in the semilinear case. The important coherence, finiteness, and determinacy hypotheses are introduced. In Sec. V the additional work for quasilinear terms is presented. The proof of stability is given in Sec. VI.

\section{TWO MODELS FROM NONLINEAR OPTICS}

The speed of propagation of light in dense materials like glass and water depends on the frequency. As a consequence white light passing through a prism is decomposed into a spectrum of colors, a discovery of Newton. These phenomena are called linear dispersion. Here the qualifier linear means that the superposition principal holds.

The development of lasers allowed the exploration of high-intensity electromagnetic waves and led to the discovery that the speed and therefore the refractive index depends also on the intensity of the field, $n=n(\omega, I)$. Taylor expansion at $I=0$ yields

$$
n(\omega, I) \sim n_{0}(\omega)+n_{2}(\omega) I+\cdots,
$$

where the notations are those standard in the physics literature. Truncating at the $n_{2}$ term yields

$$
n(\omega)=n_{0}(\omega)+n_{2}(\omega) I,
$$

which is called the Kerr nonlinearity. The common sign $n_{2}>0$ means that speed of propagation is a decreasing function of intensity of the light.

\section{A. The Lorentz model for linear dispersion}

Materials which exhibit an appreciable nonlinear index are usually dispersive in the classical sense; the speed depends on frequency. Thus the point of departure for modeling the nonlinear index are models of linear dispersion. The standard model, due to Lorentz, ${ }^{20}$ is discussed in Ref. 3. In particular its relation to excellent textbook treatments (Refs. 1 and 21) is discussed. In units normalized so that the speed of light is equal to 1 , the equations read

$$
\begin{gathered}
E_{t}=\operatorname{curl} B-P_{t}, \quad B_{t}=-\operatorname{curl} E, \quad \epsilon^{2} \partial_{t}^{2} P+P=\gamma E . \\
\operatorname{div}(E+P)=0, \quad \operatorname{div} B=0,
\end{gathered}
$$

The unknowns are the electric and magnetic fields, $E, B$, and the polarization per unit volume, $P$. The last equation from (2.2) shows that the local polarization responds to the electric field as a field of harmonic oscillators. Equation (2.3) is satisfied for all times as soon as it is satisfied at $t=0$. Thus, it is a constraint on the initial data.

The key observation is the appearance of the small parameter $\epsilon$ which has the order of magnitude of the wavelength of light divided by the next smallest characteristic length in the problem. For example for the propagation of a bullet- or cigar-shaped laser pulse through an ordinary sized glass lens, the smallest length scale is the spotsize typically of order $1 \mathrm{~mm}$, and then $\epsilon<10^{-3}$.

To convert (2.2) to a first-order system, introduce 


$$
Q:=\epsilon \partial_{t} P, \quad u:=(E, B, P, Q) .
$$

The dynamics then reads

$$
\epsilon E_{t}=\epsilon \operatorname{curl} B-\epsilon P_{t}, \quad \epsilon B_{t}=-\epsilon \operatorname{curl} E, \quad \epsilon P_{t}=Q, \quad \epsilon \partial_{t} Q+P=\gamma E .
$$

There is a natural quadratic energy. Multiplying the $E$ equation by $E$, the $B$ equation by $B$, the $P$ equation by $P$, and the $Q$ equation by $\gamma^{-1} Q$, then integrating the sum of the resulting expressions over $\mathbf{R}^{d}$ yields the conservation law

$$
0=\partial_{t} \int_{\mathbf{R}^{d}}\left\langle A_{0} u_{0}, u_{0}\right\rangle d y, \quad A_{0}:=\operatorname{diag}\left(I, I, \frac{1}{\gamma} I, \frac{1}{\gamma} I\right) .
$$

Multiplying equations (2.5) and (2.6) by $A_{0}$ expresses the fundamental system (2.2) in the form

$$
L(\epsilon \partial) u=0, \quad L(\xi):=\sum_{\mu=0}^{d} \xi_{\mu} A_{\mu}+L_{0},
$$

where the $12 \times 12$ matrices $A_{\mu}$ are real symmetric, and

$$
L_{0}=\left(\begin{array}{cccc}
0 & 0 & 0 & 1 \\
0 & 0 & 0 & 0 \\
0 & 0 & 0 & -1 / \gamma \\
-1 & 0 & 1 / \gamma & 0
\end{array}\right)
$$

is real antisymmetric. This is a dispersive symmetric hyperbolic system in the sense of Sec. III. The key ingredient in the modeling of dispersion is the fact of studying wavelength $\epsilon$ oscillations of a system in $\epsilon \partial$.

Nonlinear optical models are characterized by the fact that the polarization $P$ responds to the electric field in a nonlinear way. Two standard models are described below. For each of the nonlinear models, the linearization at $u=0$ is given by the Lorentz model. Thus the Lorentz model yields a good description of solutions of the nonlinear equations for very weak fields. The asymptotic analysis of this paper describes high-frequency solutions for which the nonlinear effects are important.

\section{B. The anharmonic oscillator model}

The change here is to model the response of the polarization as an anharmonic oscillator

$$
\epsilon^{2} P_{t t}+\nabla V(P)=\gamma E
$$

(see Refs. 22 and 23). The medium is supposed to be centrosymmetric which means that $V$ is an even function, i.e., satisfies $V(-P)=V(P)$. For low fields, the classical harmonic oscillator is a good approximation. Denote by $E_{a} \sim 10^{11} \mathrm{~m}^{-1}$ the electric field felt by an electron in a Hydrogen atom. For very high fields, i.e., $E>E_{a}, I>10^{16} \mathrm{~W} / \mathrm{cm}^{2}$, ionization processes occur and typically the materials through which the light is passing are damaged. There is an intermediate range $I \sim 10^{12} \mathrm{~W} / \mathrm{cm}^{2}$ where the intensity is low enough to avoid breakdown but high enough so that the perturbations of the harmonic oscillator have to be taken into account. This regime, sometimes called that of weak nonlinearity, is the field of nonlinear optics.

Replacing $E$ by $E / E_{a}, B$ by $c B / E_{a}$, and $P$ by $P / \epsilon_{0} E_{a}$ gives dimensionless fields. The regime of weak nonlinearity discussed above corresponds to dimensionless fields small compared to one. It is thus reasonable to introduce the Taylor expansion of the smooth real-valued potential 


$$
V(P) \sim \frac{|P|^{2}}{2}-\frac{\alpha|P|^{4}}{4}+\cdots, \quad \alpha>0 .
$$

The asymptotic analysis of Sec. IV shows that the coefficient $\alpha$ is essentially the same quantity as the Kerr Law constant $n_{2}$ and is of magnitude $O(1)$ in the nondimensional units above.

It may seem that for fields small compared to one, the nonlinear term will be negligible. However, the natural eikonal equation and polarization identities are such that the leading-order linear terms in (2.8) exactly compensate, so that the nonlinear term is crucial.

Introducing the unknown $u$ from (2.4), this model is a dispersive semilinear symmetric hyperbolic system. It is semilinear because the nonlinear $\nabla V(P)$ does not involve derivatives.

\section{Instantaneous nonlinear response} by

This model supposes that the nonlinear response of the polarization is instantaneous and given

$$
P_{N}=P_{N}(E), \quad P_{N}(-E)=-P_{N}(E), \quad \partial_{E}^{\beta} P_{N}(0)=0 \quad \text { for } \quad|\beta| \leqslant 2 .
$$

In the dimensionless units above, the fields of interest are small compared to one and the $P_{N}$ can be replaced by the leading term in its Taylor expansion

$$
P_{N}=\alpha|E|^{2} E,
$$

where the constant $\alpha \sim O(1)$ is essentially the $n_{2}$ in Kerr's Law. The polarization is the sum of this instantaneous cubic term and the term of Lorentz. The system of equations defining the dynamics is then

$$
\begin{gathered}
E_{t}=\operatorname{curl} B-\left(P+\alpha|E|^{2} E\right)_{t}, \quad B_{t}=-\operatorname{curl} E, \quad \epsilon^{2} \partial_{t}^{2} P+P=\gamma E, \\
\operatorname{div}\left(E+\alpha|E|^{2} E+P\right)=0, \quad \operatorname{div} B=0 .
\end{gathered}
$$

For the unknown $u$ as in Sec. II A, this is a dispersive symmetric hyperbolic system which is quasilinear because of the term $\left(\alpha|E|^{2} E\right)_{t}$.

\section{ASYMPTOTIC ANALYSIS OF LINEAR DISPERSIVE HYPERBOLIC SYSTEMS}

This section presents background material on dispersive symmetric hyperbolic equations. Symmetric hyperbolicity hypothesis: Suppose that

$$
L(\partial):=\sum_{\mu=0}^{d} A_{j} \frac{\partial}{\partial x_{j}}+L_{0}=A_{0} \partial_{0}+A_{0} \partial_{1}+\cdots+A_{d} \partial_{d}+L_{0} .
$$

The system of partial differential operators $L(\partial)$ is supposed to be a constant coefficient conservative symmetric hyperbolic system of order one with timelike variable $t:=x_{0}$, that is, the coeffcients $A_{\mu}$ are $N \times N$ Hermitian symmetric matrices with $A_{0}$ strictly positive and $L_{0}$ is an antiHermitian matrix.

Aside: One could consider systems with $x, \epsilon$-dependent coefficients satisfying

$$
\begin{gathered}
A_{j}(x, \boldsymbol{\epsilon})=A_{j}(x, \boldsymbol{\epsilon})^{*}, \quad A_{0}>c I>0, \\
\partial_{\epsilon, x}^{\alpha}\left\{A_{\mu}, L_{0}\right\} \in L^{\infty}\left(\mathbf{R}^{1+d}\right), \quad L_{0}(x, 0)+L_{0}^{*}(x, 0) \geqslant 0,
\end{gathered}
$$


for some constant $c$. The analysis for nonlinear phases extends without essential modification to this case. The price to pay is heavier notation. Physically, the variable coefficients represent a medium whose properties vary smoothly from point to point.

The goal is to describe oscillatory solutions of $L(\epsilon \partial) u^{\epsilon}=\left(L_{1}(\epsilon \partial)+L_{0}\right) u^{\epsilon}=0$ which have wavelength $\epsilon \ll 1$. The presence of the operators $\epsilon \partial$ makes the system singular. Exactly the same sort of singularity is familiar from the semiclassical limit in quantum mechanics. It has the peculiarity of rendering the principal part and lower-order terms in the equation of the same order. That is, the natural principal symbol involves the lower-order terms as well as the terms of order 1. The natural eikonal equation is not homogeneous in $d \phi$ and equivalently, the natural principal symbol is not homogeneous in $\xi$. This is nearly equivalent to the fact that dispersive phenomena, where speeds depend on the modulus of the wave number, can be modeled.

\section{A. Plane waves and dispersion relations}

The point of departure is the fact that linear partial differential operators with constant coefficients act simply on exponential functions of the form $e^{i \beta . x / \epsilon} \mathbf{r}$ where $\beta \in \mathbf{R}^{1+d}$ and $r \in \mathbf{C}^{N}$. Such expressions are called plane waves. One has

$$
L(\epsilon \partial)\left(e^{i \beta . x / \epsilon} \mathbf{r}\right)=e^{i \beta . x / \epsilon} L(i \beta) \mathbf{r}=e^{i \beta . x / \epsilon}\left(L_{1}(i \beta)+L_{0}\right) \mathbf{r} .
$$

There is a fundamental dichotomy. If $L(i \beta)$ is invertible, then the equation

$$
L(\epsilon \partial) u^{\epsilon}=e^{i \beta . x / \epsilon} \mathbf{b}
$$

has a unique plane wave solution

$$
u^{\epsilon}=e^{i \beta . x / \epsilon} L(i \beta)^{-1} \mathbf{b},
$$

and the homogeneous equation $L(\epsilon \partial) u=0$ has no plane wave solutions.

On the other hand, if $L(i \beta)$ is not invertible, there are plane wave solutions of the homogeneous equation, and the inhomogeneous equation has plane wave solutions only for special values of $\mathbf{b}$, those which satisfy $\pi(\beta) \mathbf{b}=0$ where $\pi(\beta)$ is defined as follows.

Definitions: The covector $\beta \in \mathbf{R}^{1+d}$ is $\epsilon$-characteristic denoted $\beta \in \epsilon \operatorname{char}(L)$ when

$$
\operatorname{det} L(i \beta)=0 .
$$

Equation (5) is called the dispersion relation. For $\beta \in \mathbf{R}^{1+d}, \pi(\beta)$ denotes the spectral projection of $L(i \beta)$ onto its kernel. Define a partial inverse $Q(\beta)$ from $\mathbf{C}^{N}$ to itself by

$$
Q(\beta) \pi(\beta)=0, \quad Q(\beta) L(i \beta) w=(I-\pi(\beta)) w \quad \text { for all } w \in \mathbf{C}^{N} .
$$

Remarks: (1) For $\beta \in \epsilon$ char, the projection $\pi(\beta)$ is equal to

$$
\pi(\beta):=\frac{1}{2 \pi i} \oint_{|z|=r}(z-L(i \beta))^{-1} d z,
$$

where $r$ is so small that 0 is the only eigenvalue inside the contour. Symmetric hyperbolicity implies that $L(i \beta)$ is an anti-Hermitian matrix, so $\pi(\beta)$ is an orthogonal projector and the algebraic and geometric multiplicity of the eigenvalue 0 are equal. Here the geometric multiplicity is defined as $\operatorname{dim} \operatorname{ker} L(i \beta)$, while the algebraic multiplicity is defined to be order of the root $z=0$ of the equation $\operatorname{det}(z I-L(i \beta))=0$.

(2) $\beta \in \mathbf{R}^{1+d} \backslash \epsilon \operatorname{char} \Rightarrow \pi(\beta)=0$.

(3) The matrix $Q$ is given by the contour integral 


$$
Q(\beta)=\frac{i}{2 \pi} \oint_{|z|=r} \frac{1}{z}(z-L(i \beta))^{-1} d z
$$

Symmetric hyperbolicity implies that $Q(\beta)$ is anti-Hermitian.

(4) For $\beta \in \epsilon$ char, $\mathbf{r}$ belongs to the kernel of $L(i \beta)$ if and only if $u^{\epsilon}:=e^{i \beta . x / \epsilon} \mathbf{r}$ are plane wave solutions of the equation $L(\epsilon \partial) u^{\epsilon}=0$. The equation (3) has a plane wave solution if and only if $\pi(\beta) \mathbf{b}=0$, in which case the solutions are given by the vectors $\mathbf{r}$ satisfying

$$
\mathbf{r}-Q(\beta) \mathbf{b} \in \operatorname{ker} L(i \beta) .
$$

Proposition 3.1: For each $\eta \in \mathbf{R}^{d}$ there are at most $N$ values $\tau$ such that $(\tau, \eta) \in \epsilon$ char. If $\tau_{1}<\tau_{2}<\cdots<\tau_{k}$ are the values, then

$$
\mathbf{C}^{N}=\sum_{m=1}^{k} \operatorname{ker} L\left(i \tau_{m}, i \eta\right)
$$

is an orthogonal decomposition with respect to the scalar product determined by $A_{0}$.

Proof: Since $L(i \beta)=i\left(L_{1}(\beta)-i L_{0}\right)$, it follows that $\beta:=(\tau, \eta)$ belongs to $\epsilon$ char if and only if $\tau$ satisfies

$$
\operatorname{det}\left(\tau A_{0}+\sum_{j=0}^{d} \eta_{j} A_{j}-i L_{0}\right)=0
$$

Since $A_{0}$ is positive definite and $\sum_{j=0}^{d} \eta_{j} A_{j}-i L_{0}$ is Hermitian symmetric, the result follows from the spectral theorem.

It follows that for $\mathbf{r} \in \mathbf{C}^{N}$ the initial value problem

$$
L(\epsilon \partial) u^{\epsilon}=0,\left.\quad u^{\epsilon}\right|_{t=0}=e^{i \eta \cdot y / \epsilon} \mathbf{r}
$$

has the exact solution

$$
u^{\epsilon}:=\sum_{m=1}^{k} e^{i\left(\tau_{m}, \eta\right) \cdot x / \epsilon} \mathbf{r}_{m}, \quad \text { where } \quad \mathbf{r}=\sum_{0}^{k} \mathbf{r}_{m}
$$

is the decomposition of $\mathbf{r}$ into elements of $\operatorname{ker} L\left(i \tau_{m}, i \eta\right)$.

In the Proposition the scalar product with respect to $A_{0}$ plays an important role. This is tied to the fact that the time variable $t$ plays a distinguished role in the decomposition $\beta=(\tau, \eta)$. For a different time variable the orthogonality of the kernels would be with respect to a different scalar product. The projectors $\pi(\beta)$ on the kernel are chosen orthogonal with respect to the scalar product in $\mathbf{C}^{N}$ which is the scalar product for which the symbol $L(i \beta)$ is anti-Hermitian.

Example: For the Klein-Gordon operator $L(\epsilon \partial)=\epsilon^{2} \square+1, \beta \in \epsilon$ char if and only if it satisfies the dispersion relation $\beta_{0}^{2}=\beta_{1}^{2}+\cdots+\beta_{d}^{2}+1$. This is a second-order analogue, studied by seeking plane wave solutions $u^{\epsilon}=e^{i \beta . x / \epsilon} c$. The identity

$$
\left(\epsilon^{2} \square+1\right) u^{\epsilon}=e^{i \beta . x / \epsilon}\left(-\beta_{0}^{2}+\beta_{1}^{2}+\cdots+\beta_{d}+1\right)
$$

yields the dispersion relation.

Example: Compute plane wave solutions of the Lorentz model from Sec. II A by seeking exact solutions of the form $e^{\beta . x / \epsilon}(\mathbf{e}, \mathbf{b}, \mathbf{p}, \mathbf{q})$. This yields the system of homogeneous linear equations

$$
i \tau \mathbf{e}-i \eta \wedge \mathbf{b}=-\mathbf{q}, \quad \tau \mathbf{b}+\eta \wedge \mathbf{e}=0, \quad i \tau \mathbf{p}=\mathbf{q}, \quad i \tau \mathbf{q}=-\mathbf{p}+\gamma \mathbf{e} .
$$


Fix $\eta$ and seek the values $\tau=\tau(\eta)$ belonging to the variety.

There are always solutions with $\tau=0$. The corresponding vectors are those with

$$
\mathbf{e}\|\eta, \quad \mathbf{b}\| \eta, \quad \mathbf{p}=\gamma \mathbf{e}, \quad \mathbf{q}=0
$$

This is a two-dimensional family. None of the nonzero elements of this family satisfy the constraint (2.5).

For $\tau \neq 0$ one can eliminate $\mathbf{b}$ and $\mathbf{q}$ from the system to obtain the reduced system

$$
\tau^{2} \mathbf{e}+\eta \wedge(\eta \wedge \mathbf{e})=-\tau^{2} \mathbf{p}, \quad\left(-\tau^{2}+1\right) \mathbf{p}=\gamma \mathbf{e}
$$

In the first equation, simplify the double cross product and multiply by $\tau^{2}-1$ to eliminate $\mathbf{p}$ to find

$$
\left(\left(\tau^{2}-1\right)\left(\tau^{2}-\left|\eta^{2}\right|+|\eta\rangle\langle\eta|\right)-\gamma \tau^{2}\right) \mathbf{e}=0
$$

The physically relevant solutions satisfy the constraint $\eta \cdot \mathbf{e}=0$, which yields the dispersion relation

$$
0=\left(\tau^{2}-1\right)\left(\tau^{2}-\left|\eta^{2}\right|\right)-\gamma \tau^{2}=\tau^{4}-\left(1+\gamma+|\eta|^{2}\right) \tau^{2}+|\eta|^{2} .
$$

Note that this equation is not homogeneous in $\tau, \eta$. The spectral projection $\pi(\beta)$ corresponding to the roots of (3.11) is orthogonal projection on the kernel which is the set of vectors satisfying

$$
\mathbf{e} \perp \eta, \quad \tau \mathbf{b}=-\eta \wedge \mathbf{e}, \quad \mathbf{p}=\frac{\gamma}{-\tau^{2}+1} \mathbf{e}, \quad \mathbf{q}=i \tau \mathbf{p} .
$$

Note that the phase velocity $v_{\phi}=-\tau \eta /|\eta|^{2}$ so that the triple $v_{\phi}, \mathbf{e}, \mathbf{b}$ is an oriented triple in $\mathbf{R}^{3}$. Equation (3.13) defines a kernel of dimension two parametrized by the vectors $\mathbf{e} \perp \eta$ by the mapping

$$
\mathbf{e} \perp \eta \mapsto K(\mathbf{e}):=\left(\mathbf{e}, \frac{-\eta}{\tau} \wedge \mathbf{e}, \frac{\gamma}{-\tau^{2}+1} \mathbf{e}, \frac{i \tau \gamma}{-\tau^{2}+1} \mathbf{e}\right)
$$

Note that for $\mathbf{e} \perp \eta$,

$$
\|K(\mathbf{e})\|=\nu\|\mathbf{e}\|, \quad \nu(\tau, \eta):=\left(1+\frac{\eta^{2}}{\tau^{2}}+\frac{\gamma^{2}\left(1+\tau^{2}\right)}{\left(1-\tau^{2}\right)^{2}}\right)^{1 / 2}
$$

Set

$$
S(u)=S(E, B, P, Q):=E+\frac{\eta}{\tau} \wedge B+\frac{\gamma}{-\tau^{2}+1} P-\frac{i \tau \gamma}{-\tau^{2}+1} Q
$$

We next show that

$$
\nu^{2} \pi(u)=K\left(p_{\eta \perp}(S(u))\right)=K\left(S(u)-\frac{\langle S(u), \eta\rangle \eta}{\|\eta\|^{2}}\right) .
$$

Since both sides are linear it suffices to prove (3.14) for $u$ either belonging to or orthogonal to ker $\pi((u)$. We use two identities satisfied for all $\mathbf{e} \perp \eta$.

$$
(u, K(\epsilon))=(S(u), \mathbf{e}) \quad \text { and } \quad S(K(\mathbf{e}))=\nu^{2} \mathbf{e}
$$


The first identity shows that $\pi(u)=0$ if and only if $p_{\eta \perp}(S(u))=0$, so (3.14) holds for $u \in \operatorname{ker} \pi$. For $0 \neq u \perp$ ker $\pi$, choose $\mathbf{e} \perp \eta$ so that $u=K(\mathbf{e})$. Then

$$
K\left(p_{\nu \perp}(S(u))\right)=K\left(p_{\eta \perp}(S(K(e)))\right)=K\left(\left(p_{\eta \perp}\left(\nu^{2} \mathbf{e}\right)\right)\right)=K\left(\nu^{2} e\right)=\nu^{2} u
$$

verifying (3.14) in these cases and therefore completing the proof of (3.14).

The dispersion relation yields a quadratic equation for $\tau^{2}$ which is explicitly solvable. The resulting expression is rarely used. The simple explicit representation

$$
|\eta|^{2}=\tau^{2}\left(1-\frac{\gamma}{\tau^{2}-1}\right)
$$

for $\eta$ as a function of $\tau$ is often preferred.

The remaining solutions of the homogeneous linear system are those with $e \| \eta$ which in addition to those with $\tau=0$ yield a family with $\tau^{2}-1-\gamma=0$. This inventory of 12 solutions $\tau$ for each $\eta$ shows that equation (3.5) is exactly

$$
\tau^{2}\left(\tau^{2}-1-\gamma\right)\left[\left(\tau^{2}-1\right)\left(\tau^{2}-\left|\eta^{2}\right|\right)-\gamma \tau^{2}\right]^{2}=0 .
$$

\section{B. Approximate solutions with varying amplitude and linear phases}

We construct solutions which on scales $o$ (1) look like plane waves, but whose amplitudes are smoothly varying on scales $O(1)$. The residuals are infinitely small. Seek asymptotic solutions of the equation

$$
L(\epsilon \partial) u^{\epsilon}=\left(L_{1}(\epsilon \partial)+L_{0}\right) u^{\epsilon}=b(x, \epsilon) e^{i \beta . x / \epsilon}, \quad b(x, \epsilon) \sim \sum_{j=0}^{\infty} \epsilon^{j} b_{j}(x),
$$

with

$$
u^{\epsilon}=e^{i \beta . x / \epsilon} a(x, \epsilon), \quad a(x, \epsilon) \sim \sum_{j=0}^{\infty} \epsilon^{j} a_{j}(x) .
$$

Plugging $u^{\epsilon}$ into the partial differential operator yields

$$
L(\epsilon \partial) u^{\epsilon}=e^{i \beta . x / \epsilon}\left(L_{1}(\epsilon \partial) a+L(i \beta) a\right) .
$$

The strategy is to expand

$$
L(i \beta) a+L_{1}(\epsilon \partial) a-b \sim \sum_{j=0}^{\infty} \epsilon^{j} c_{j}(x),
$$

and to choose the coefficients $a_{j}$ so that all the $c_{j}$ vanish identically.

Setting the leading term in (3.19) equal to zero yields

$$
0=L(i \beta) a_{0}-b_{0} .
$$

There is a fundamental dichotomy. If $L(i \beta)$ is nonsingular, then $a_{0}$ is determined from $b_{0}$ by the linear algebraic equation (3.20). An entire asymptotic expansion is determined by such local algebraic equations. This is in exact analogy to elliptic high-frequency asymptotics (see Refs. 24 and 25).

The situation is more interesting when $L(i \beta)$ is singular. In that case, multiplying (3.20) by $\pi(\beta)$ yields the constraint 


$$
\pi(\beta) b_{0}=0
$$

Multiply (3.20) by $Q(\beta)$ to find

$$
(I-\pi(\beta)) a_{0}=Q(\beta) b_{0}
$$

Equation (3.20) holds if and only if (3.21) and (3.22) hold.

Setting $c_{1}=0$ in (3.19) yields

$$
\left(L_{0}+L_{1}(i \beta)\right) a_{1}+L_{1}(\partial) a_{0}=b_{1} \text {. }
$$

Typical of two scale expansions, this equation involves coefficients $a_{0}$ and $a_{1}$ of two different powers of $\epsilon$. Multiplying by $\pi(\beta)$ eliminates the $a_{1}$ term. This yields

$$
\pi(\beta) b_{1}=\pi(\beta) L_{1}(\partial) a_{0}=\pi(\beta) L_{1}(\partial) \pi(\beta) a_{0}+\pi(\beta) L_{1}(\partial)(I-\pi(\beta)) a_{0}
$$

Using (3.22) gives

$$
\pi(\beta) L_{1}(\partial) \pi(\beta) a_{0}=\pi(\beta) b_{1}-\pi(\beta) L_{1}(\partial) Q(\beta) b_{0}
$$

Equation (3.23) yields an initial value problem for $\pi(\beta) a_{0}$ involving the first-order partial differential operator $\pi(\beta) L_{1}(\partial) \pi(\beta)$. That this problem is solvable is guaranteed by the following simple but important Proposition.

Proposition 3.2: If $L_{1}\left(\partial_{x}\right)$ is a symmetric hyperbolic operator and $0 \neq \pi \in \operatorname{Hom}\left(\mathbf{C}^{N}\right)$ is an orthogonal projector, then $\pi L_{1}\left(\partial_{x}\right) \pi$ is symmetric hyperbolic operator acting on functions with values in Range $\pi$.

Proof: The coefficient of $\partial_{j}$ is equal to the restriction of $\pi A_{j} \pi$ to Range $\pi$ which is Hermitian since $A_{j}$ and $\pi$ are. is.

The coefficient of $\partial_{0}=\partial_{t}$ is the restriction of $\pi A_{0} \pi$ to Range $\pi$ so is positive definite since $A_{0}$

Thus, equation (3.23) is a linear symmetric hyperbolic system and so determines $\pi(\beta) a_{0}$ from its initial data. Since $(I-\pi(\beta)) a_{0}$ is already known, this completes the determination of $a_{0}$. If $\pi(\beta)$ has rank one, then $\pi(\beta) L_{1}(\partial) \pi(\beta)$ is a directional derivative in the direction of a constant coefficient vector field. Such transport operators also can arise when $\pi(\beta)$ has rank greater than one. When $\pi(\beta) L_{1}(\partial) \pi(\beta)$ is a directional derivative, $\pi(\beta) a_{0}$ is determined by solving ordinary differential equations along the integral curves of the vector field. The classic example which leads to an operator which is not a directional derivative is conical refraction. In Proposition 3.3 we show that this exceptional behavior occurs only for points $\beta$ where two or more sheets of the $\epsilon$-characteristic variety intersect.

Example: For the Klein-Gordon operator $\epsilon^{2} \square+1$, suppose that $\beta$ satisfies the dispersion relation $\beta_{0}^{2}=\beta_{1}^{2}+\cdots+\beta_{d}^{2}+1$. Then

$$
\left(\epsilon^{2} \square+1\right) u e^{\epsilon} \sim e^{i \beta \cdot x / \epsilon}\left(\epsilon 2 i\left(\beta_{0} \partial_{t}-\sum_{j=0}^{d} \beta_{j} \partial_{j}\right) a_{0}+O\left(\epsilon^{2}\right)\right) .
$$

This yields the transport equation

$$
\left(\beta_{0} \partial_{t}-\sum_{j=0}^{d} \beta_{j} \partial_{j}\right) a_{0}=0
$$

The amplitude $a_{0}$ is rigidly transported with velocity given by 


$$
\mathbf{v}_{g}:=\text { group velocity: }=\frac{-\left(\beta_{1}, \ldots, \beta_{d}\right)}{\beta_{0}} .
$$

For comparison, the phase velocity is given by

$$
v_{\phi}:=\text { phase velocity }=\frac{-\beta_{0}\left(\beta_{1}, \ldots, \beta_{d}\right)}{\left(\beta_{1}^{2}+\cdots+\beta_{d}^{2}\right)} .
$$

The appearance of phase velocities which depend on $|\beta|$ is the signature of dispersion.

The next proposition gives a sufficient condition for the symmetric hyperbolic operator $\pi(\beta) L_{1}(\beta) \pi(\beta)$ to be a simple directional derivative.

Proposition 3.3: Suppose that the $\epsilon$-characteristic variety of $L$ is a graph near $\beta=(\tau, \eta) \in \mathbf{R}^{1+d}$ in the sense that for $\eta$ near $\eta$ there is a unique point $(\tau(\eta), \eta) \in \epsilon$ char near $\beta$. Then $\tau(\eta)$ is a smooth function of $\eta$ and if one defines the transport operator at the group velocity by

$$
V\left(\underline{\beta} ; \partial_{x}\right):=\frac{\partial}{\partial t}-\sum_{j=0}^{d} \frac{\partial \tau(\underline{\eta})}{\partial \eta_{j}} \frac{\partial}{\partial y_{j}}:=\frac{\partial}{\partial t}+\mathbf{v}_{g} . \partial_{y},
$$

then $\pi(\beta) L_{1}(\partial) \pi(\beta)$ is the simple directional derivative

$$
\pi(\underline{\beta}) L_{1}\left(\partial_{x}\right) \pi(\underline{\beta})=\pi(\underline{\beta}) A_{0} \pi(\underline{\beta}) V\left(\partial_{x}\right) .
$$

Proof: Since

$$
L(i \beta)=A_{0}^{1 / 2}\left(i \tau I+\sum_{j=1}^{d} i \eta_{j} A_{0}^{-1 / 2} A_{j} A_{0}^{-1 / 2}+A_{0}^{-1 / 2} L_{0} A_{0}^{-1 / 2}\right) A_{0}^{1 / 2},
$$

the solutions $\tau$ are the eigenvalues of the Hermitian matrix

$$
H(\eta):=A_{0}^{-1 / 2}\left(i L_{0}-\sum_{j=0}^{d} \eta_{j} A_{j}\right) A_{0}^{-1 / 2}
$$

Choose $r>0$ so that for $\eta$ near $\eta$ there is exactly one eigenvalue in the disk of center $\beta_{0}$ and radius $r$. The smoothness of $\tau(\eta)$ then follows from the contour integral representation

$$
\tau(\eta)=\frac{\operatorname{Trace}\left(\frac{1}{2 \pi} \oint_{|z|=r} z(z I-H(\eta))^{-1} d z\right)}{\operatorname{Trace}\left(\frac{1}{2 \pi} \oint_{|z|=r}(z I-H(\eta))^{-1} d z\right)} .
$$

Differentiate the identity $L(i \tau(\eta), i \eta) \pi(\tau(\eta), \eta)=0$ with respect to $\eta_{j}$ to find

$$
L(i \tau(\eta), \eta) \frac{\partial}{\partial \eta_{j}} \pi(\tau(\eta), \eta)+i\left(\frac{\partial \tau(\eta)}{\partial \eta_{j}} A_{0}+A_{j}\right) \pi(\tau(\eta), \eta)=0 .
$$

Multiplying by $\pi(\tau(\eta), \eta)$ eliminates the first term to give

$$
\pi(\tau(\eta), \eta)\left(\frac{\partial \tau(\eta)}{\partial \eta_{j}} A_{0}+A_{j}\right) \pi(\tau(\eta), \eta)=0 .
$$


Using this for the summands on the right-hand side of the identity

$$
\pi(\beta) L_{1}\left(\partial_{x}\right) \pi(\beta)=\pi(\beta) A_{0} \pi(\beta) \frac{\partial}{\partial t}+\sum_{j=1}^{d} \pi(\beta) A_{j} \pi(\beta) \frac{\partial}{\partial x_{j}}
$$

yields the desired relation

$$
\pi(\beta) L_{1}\left(\partial_{x}\right) \pi(\beta)=\pi(\beta) A_{0} \pi(\beta)\left(\frac{\partial}{\partial t}-\sum_{j=0}^{d} \frac{\partial \tau(\eta)}{\partial \eta_{j}} \frac{\partial}{\partial x_{j}}\right)
$$

Example: For the Lorentz model consider $\eta \neq 0$ and $\beta=(\tau, \eta) \in \epsilon$ char with $\tau^{2} \notin\{0,1+\gamma\}$. Then $\tau^{2}$ satisfies (3.13) which has a pair of distinct positive solutions for each $\eta$ since

$$
\text { discriminant }=\left(|\eta|^{2}+1+\gamma\right)^{2}-4|\eta|^{2}=\left(|\eta|^{2}+(\gamma-1)\right)^{2}-(\gamma-1)^{2}+(1+\gamma)^{2}>0 .
$$

Equation (3.15) shows that the graph of $|\eta|^{2}$ as a function of $\tau^{2}$ rises from 0 to $\infty$ as $\tau^{2}$ increases from 0 to 1 . There are no solutions with $\tau^{2}$ between 1 and $1+\gamma$. The graph then rises from 0 to $\infty$ approaching the line $|\eta|^{2}=\tau^{2}$ from below as $\tau^{2} \rightarrow \infty$. The slope is always positive in fact,

$$
\frac{d|\eta|^{2}}{d \tau^{2}}=1+\frac{\gamma}{\left(\tau^{2}-1\right)^{2}}>0
$$

The group velocity is computed as follows. The chain rule yields

$$
\frac{d|\tau|}{d|\eta|}=\frac{d|\tau|}{d \tau^{2}} \frac{d \tau^{2}}{d|\eta|^{2}} \frac{d|\eta|^{2}}{d|\eta|}=\left(\frac{d \tau^{2}}{d|\tau|}\right)^{-1}\left(\frac{d|\eta|^{2}}{d \tau^{2}}\right)^{-1} 2|\eta|=\frac{1}{2|\tau|}\left(1+\frac{\gamma}{\left(\tau^{2}-1\right)^{2}}\right)^{-1} 2|\eta|
$$

This yields

$$
\text { group velocity: }=\mathbf{v}_{g}(\tau, \eta)=-\nabla_{\eta} \tau(\eta)=-\frac{\eta}{\tau}\left(1+\frac{\gamma}{\left(\tau^{2}-1\right)^{2}}\right)^{-1} .
$$

The transport operator simplifies a little. Using (2.6) and (3.14) one shows that

$$
\pi(\tau, \eta) A_{0} \pi(\tau, \eta)=\mu(\tau, \eta) \pi(\tau, \eta)
$$

where

$$
\mu(\tau, \eta):=\left(1+\frac{\eta^{2}}{\tau^{2}}+\frac{\gamma\left(1+\tau^{2}\right)}{\left(1-\tau^{2}\right)^{2}}\right) /\left(1+\frac{\eta^{2}}{\tau^{2}}+\frac{\gamma^{2}\left(1+\tau^{2}\right)}{\left(1-\tau^{2}\right)^{2}}\right)=2 / v_{g} \cdot v_{\phi}\left(1+\frac{\eta^{2}}{\tau^{2}}+\frac{\gamma^{2}\left(1+\tau^{2}\right)}{\left(1-\tau^{2}\right)^{2}}\right)
$$

It follows that for linear phases the transport equation is simply

$$
\left(\partial_{t}+v_{g} \cdot \partial_{y}\right) \mathbf{e}=0
$$

where the $\mathbf{b}, \mathbf{p}, \mathbf{q}$ components of $a_{0}$ are computed from $\mathbf{e}$ using (3.13).

The next Proposition shows that whether or not the operator $\pi L_{1}(\partial) \pi$ is a simple transport operator, its sound speeds are always between the smallest and largest sound speeds of $L_{1}(\partial)$. Recall that the sound speeds $c(\eta)$ defined for unit vectors $\eta \in \mathbf{R}^{d}$ are the roots of $\operatorname{det} L_{1}(-c, \eta)$ (see Ref. 26). Note that only the principal part of $L$ is involved in this definition, in contrast to the definition of $\epsilon$ char and the group velocities of $L(\epsilon \partial)$.

Proposition 3.4: If $L_{1}(\partial)$ is a symmetric hyperbolic operator and $\pi \in \operatorname{Hom}\left(\mathbf{C}^{N}\right) \backslash 0$ is an orthogonal projector, then the sound speeds of the symmetric hyperbolic operator $\pi L_{1}(\partial) \pi$ defined 
on Range $\pi$-valued functions lie between the largest and smallest sound speeds of $L_{1}(\partial)$. In particular, when the hypotheses of Proposition 3.3 are satisfied, the group velocities are no larger than the largest sound speed of $L_{1}$.

Proof: The sound speeds of $L_{1}(\partial)$ are the eigenvalues of the matrix $H:=-\Sigma \eta_{j} A_{j}$ with respect to the positive matrix $A_{0}$. They are the critical values of the Rayleigh quotient

$$
\frac{\langle H v, v\rangle}{\left\langle A_{0} v, v\right\rangle} .
$$

The sound speeds of the system $\pi L_{1}(\partial) \pi$ are the critical values of the same function restricted to the subspace Range $\pi$. Therefore they lie between the maximum and minimum of the Rayleigh quotient defined on all vectors. These extrema are exactly the largest and smallest speeds of the original system, which proves the result.

Even when the hypotheses of Proposition 3.3 are not satisfied, this proposition shows that the domains of influence for the reduced operators $\pi L \pi$ are always contained in the convex domain of influence of $L_{1}$.

Returning to the construction of asymptotic solutions, the coefficient $c_{j}$ with $j \geqslant 2$ is given by

$$
c_{j}(x)=\left(L_{0}+L_{1}(i \beta)\right) a_{j}+L_{1}(\partial) a_{j-1}-b_{j} .
$$

Once $a_{0}, \ldots, a_{j-1}$ are determined, the coefficient $a_{j}$ is determined from the equations $Q(\beta) c_{j}=0$ and $\pi(\beta) c_{j+1}=0$. The former implies that

$$
(I-\pi(\beta)) a_{j}=Q(\beta)\left(b_{j}-L_{1}(\partial) a_{j-1}\right),
$$

which determines $(I-\pi(\beta)) a_{j}$ from terms already known. The latter yields an evolution equation for $\pi(\beta) a_{j}$,

$$
\pi(\beta) L_{1}(\partial) \pi(\beta) a_{j}=-\pi(\beta) L_{1}(\partial) Q(\beta)\left(b_{j}-L_{1}(\partial) a_{j-1}\right)+\pi(\beta) b_{j},
$$

which serves to determine $\pi(\beta) a_{j}$ uniquely from its initial values at $\{t=0\}$. This completes the determination of the amplitudes $a_{j}$ of the formal asymptotic solution from the initial values of $\pi(\beta) a_{j \mid t=0}$ provided that the constraint (3.21) is satisfied. These computations prove the first two of the following three fundamental theorems.

Theorem 3.5: Suppose that $T>0$, that $a(\epsilon, x)$ and $b(\epsilon, x)$ belong to $\left.\left.C^{\infty}(] 0,1\right] \times[0, T] \times \mathbf{R}^{d}\right)$ and have space-time support in an $\epsilon$-independent compact subset of $[0, T] \times \mathbf{R}^{d}$ and satisfy

$$
a(\epsilon, x) \sim \sum_{0}^{\infty} \epsilon^{j} a_{j}(x) \quad \text { and } \quad b(\epsilon, x) \sim \sum_{0}^{\infty} \epsilon^{j} b_{j}(x),
$$

in the sense that $a_{j}$ and $b_{j}$ belong to $C_{(0)}^{\infty}\left([0, T] \times \mathbf{R}^{d}\right)$ and

$$
\left.\left.\forall \alpha \in \mathbf{N}^{d}, \forall m \in \mathbf{N}, \exists C, \forall \epsilon \in\right] 0,1\right], \forall x \in[0, T] \times \mathbf{R}^{d}:\left|\partial_{x}^{\alpha}\left(a(\epsilon, x)-\sum_{j=0}^{m} \epsilon^{j} a_{j}(x)\right)\right| \leqslant C \epsilon^{m+1},
$$

with a similar expression for $b(\epsilon, x)$. For $\epsilon \in] 0,1]$ define

$$
u^{\epsilon}(x):=e^{i \beta . x / \epsilon} a(\epsilon, x) .
$$

Then

$$
L(\epsilon \partial) u^{\epsilon}-e^{i \beta . x / \epsilon} b(\epsilon, x) \sim 0 \quad \text { in } C_{(0)}^{\infty}\left([0, T] \times \mathbf{R}^{d}\right)
$$


if (3.21)-(3.23) and the sequence of equations (3.25) and (3.26) are satisfied.

Theorem 3.6: Given

$$
g_{j}(y) \in C_{0}^{\infty}\left(\mathbf{R}^{d}\right) \quad \text { and } \quad b_{j} \in C_{(0)}^{\infty}\left([0, T] \times \mathbf{R}^{d}\right)
$$

with supports in a fixed compact set and satisfying

$$
\pi(\beta) g_{j}=g_{j} \quad \text { and } \quad \pi(\beta) b_{0}=0,
$$

there are uniquely determined functions $a_{j}(x) \in C_{(0)}^{\infty}\left([0, T] \times \mathbf{R}^{d}\right)$ satisfying (3.22) and (3.23), the sequence of equations (3.25) and (3.26), and the initial conditions

$$
\pi(\beta) a_{j}(0, y)=g_{j}(y) .
$$

Borel's theorem guarantees that for $a_{j}, b_{j}$ as in Theorem 3.6, there exist $a(\epsilon, x)$ and $b(\epsilon, x)$ as in Theorem 3.5, which constructs approximate solutions given by (3.29).

Theorem 3.7: Suppose that $u^{\epsilon}$ is defined as in Theorem 3.5, and that $\left.\left.v(\epsilon, x) \in C^{\infty}(] 0,1\right] \times[0, T] \times \mathbf{R}^{d}\right)$, with $\operatorname{supp} v(\epsilon,$.$) contained in an \epsilon$-independent compact set, satisfies

$$
L(\epsilon \partial) v(\epsilon, x)-e^{i \beta . x / \epsilon} b(\epsilon, x) \sim 0 \quad \text { and } \quad v(\epsilon, 0, y)-u^{\epsilon}(0, y) \sim 0
$$

in $C_{(0)}^{\infty}\left([0, T] \times \mathbf{R}^{d}\right)$ and $C_{0}^{\infty}\left(\mathbf{R}^{d}\right)$, respectively. Then

$$
v(\epsilon, x)-u^{\epsilon}(x) \sim 0 \quad \text { in } \quad C_{(0)}^{\infty}\left([0, T] \times \mathbf{R}^{d}\right) .
$$

An important case is when the right-hand sides of (3.32) and (3.33) vanish in which case $v$ is the exact solution of the problem to which $u^{\epsilon}$ is an approximate solution.

Proof: The difference $u^{\epsilon}-u_{\text {exact }}^{\epsilon}$ satisfies $L\left(u^{\epsilon}-v(\epsilon \cdot x)\right)=O\left(\epsilon^{\infty}\right)$. To show that the difference is small, one needs to estimate how fast solutions of $L u=0$ can grow.

There is subtlety here because of the $\epsilon$ in front of the $\partial_{t}$. Solutions of the equation $\left(\epsilon \partial_{t}-1\right) u=0$ are multiples of $e^{t / \epsilon}$ so grow very rapidly. If this happened in our problem, the fact that the residuals are $O\left(\epsilon^{\infty}\right)$ would be more than compensated by this exponential explosion. It is to avoid this that we assumed the conservation hypothesis $L_{0}+L_{0}^{*}=0$. This hypothesis shows that $\int\left\langle A_{0} u, u\right\rangle d x_{1} \cdots d x_{d}$ is independent of $x_{0}$ for solutions of $L u=0$.

With this hypothesis, the basic energy identity reads

$$
\sum_{\mu=0}^{d} \epsilon \partial_{\mu}\left\langle A_{\mu} u, u\right\rangle=2 \operatorname{Re}\langle L(\epsilon \partial) u, u\rangle .
$$

This implies immediately that for any $T$ and $s$ there is a constant $C$ so that for all $w \in C_{(0)}^{\infty}\left([0, T] \times \mathbf{R}^{d}\right)$

$$
\|w\|_{H^{s}\left([0, T] \times \mathbf{R}^{d}\right)} \leqslant C\left(\|w(0)\|_{\mid H^{s}\left(\mathbf{R}^{d}\right)}+\frac{1}{\epsilon}\|L(\epsilon \partial) w\|_{H^{s}\left([0, T] \times \mathbf{R}^{d}\right)}\right) .
$$

Following the work of $\operatorname{Lax}^{19}$ on the nondispersive case, apply this estimate to

$$
w:=u^{\epsilon}-v(\epsilon, x) .
$$

The potentially explosive factor $1 / \epsilon$ is absorbed by the infinitely small residuals and one obtains

$$
\|w\|_{H^{s}\left([0, T] \times \mathbf{R}^{d}\right)} \leqslant C(s, m) \epsilon^{m}
$$


for all $m$. Sobolev's Theorem completes the proof.

Example: The conservation hypothesis is crucial for Theorem 3.7. Two examples for which it is not satisfied are the Klein-Gordon operator with negative coefficient

$$
L\left(\epsilon \partial_{x}\right)=\epsilon^{2} \square-1
$$

for which the natural conserved energy has indefinite density $\epsilon^{2} u_{t}^{2}+\epsilon^{2} u_{y}^{2}-u^{2}$ and the first order system analogue

$$
L\left(\epsilon \partial_{x}\right)=\partial_{t}+\left(\begin{array}{cc}
1 & 0 \\
0 & -1
\end{array}\right) \partial_{y}+\left(\begin{array}{ll}
0 & 1 \\
1 & 0
\end{array}\right)
$$

For each of these the $\epsilon$-characteristic equation is $\tau^{2}=\eta^{2}-1$. For $|\eta|>1$ there are nice plane waves and one can construct approximate solutions

$$
u^{\epsilon}=e^{i(\tau t+\eta y) / \epsilon}\left(a_{0}(x)+\epsilon a_{1}(x)+\cdots\right), \quad L\left(\epsilon \partial_{x}\right) u^{\epsilon} \sim 0
$$

whose profiles are determined by transport equations along group lines whose velocity is given by

$$
v_{g}:=-\partial_{\eta} \tau=\frac{-\eta}{\left(\eta^{2}-1\right)^{1 / 2}}
$$

with $\left|v_{g}\right|>1$. In particular the principal profile is given by the explicit relation $a_{0}(t, y)=a_{0}\left(0, y-v_{g} t\right)$.

If the initial data for the $\pi(\beta) a_{j}$ are supported in $|y|<r$, then the profiles are supported in the tube $\left|y-v_{g} t\right|<r$ and the solution $u^{\epsilon}$ can also be taken with support in this tube. The sound speeds of the operators $L$ are \pm 1 , so that for all $\epsilon \neq 0$

$$
\operatorname{supp} v^{\epsilon} \subset\{|y| \leqslant r+|t|\} .
$$

For $|t|>2 r /\left(\left|v_{g}\right|-1\right)$ the supports of the exact and approximate solutions are disjoint!

This apparent contradiction is resolved by noting that the analogue of Theorem 3.8 is not true. The infinitely small residual cannot be removed. The family $L(\epsilon \partial)$ is not uniformly stable, so though the residuals are infinitely small the error is not. The apparently infinitely accurate approximate solutions are worthless!

\section{Nonlinear phases}

It is not difficult to extend the analysis to nonlinear phases $\phi(x)$ so that the oscillating factor is equal to $e^{i \phi(x) / \epsilon}$. To do this requires the introduction of a few additional concepts.

The eikonal equation becomes

$$
\operatorname{det} L(i d \phi(x))=0 \text {. }
$$

This asserts that for all $x, d \phi(x) \in \epsilon$ char. In the case of the Klein-Gordon equation, the eikonal equation is

$$
\phi_{t}^{2}=\left|\phi_{y}\right|^{2}+1 .
$$

For the Lorentz model, the interesting oscillations have phases satisfying the eikonal equation

$$
\left(\phi_{t}^{2}-1\right)\left(\phi_{t}^{2}-\left|\phi_{y}\right|^{2}\right)+\gamma \phi_{t}^{2}=0 .
$$


In both cases, given initial values $\phi(0, y)$ with $\phi_{y}(0, y) \neq 0$ and a value $\phi_{t}(0, y)$ satisfying the equation at $(0, y)$, there is a unique smooth local solution $\phi$. To verify this it suffices to show that the initial value problem is noncharacteristic. For the Klein-Gordon equation compute

$$
\frac{\partial\left(\phi_{t}^{2}-\left|\phi_{y}\right|^{2}-1\right)}{\partial \phi_{t}}=2 \phi_{t}= \pm 2 \sqrt{1+\left|\phi_{y}\right|^{2}} \neq 0 .
$$

For the Lorentz model, the noncharacteristic condition is equivalent to the nonvanishing of the discriminant computed after Proposition 3.3.

There are several things to notice. First, as is usual in the theory of first order partial differential equations, the time derivative must be given at one point. Second, using the determinant in (3.35), the eikonal equation that one finds is

$$
\left(\left(\phi_{t}^{2}-1\right)\left(\phi_{t}^{2}-\left|\phi_{y}\right|^{2}\right)+\gamma \phi_{t}^{2}\right)^{2} \phi_{t}^{2}\left(\phi_{t}^{2}-1-\gamma\right)=0 .
$$

For this equation, the surface $\{t=0\}$ is characteristic for any $\phi$ satisfying (3.33), because the square of the equation appears as a factor. For many problems of mathematical physics, one has characteristics of high multiplicity for which the characteristic equation is reducible, and to construct solutions of the eikonal equation, one studies the factors and not the expression of the full determinant.

The eikonal equation being nonlinear, solutions are often only locally defined. Thus the computations of the previous section must be performed locally.

Eikonal phase hypothesis: $\Omega \subset \mathbf{R}^{1+d}$ is open and the phase $\phi \in C^{\infty}(\Omega ; \mathbf{R})$ satisfies the eikonal equation (3.35), has nonvanishing differential, and $\pi(d \phi(x))$ is a smooth function on $\Omega$.

It follows that

$$
Q(d \phi(x))=(I-\pi(d \phi(x)))(\pi(d \phi(x))+L(i d \phi(x)))^{-1}
$$

is also smooth. The analysis for linear phases $\beta . x, \beta \in \epsilon$ char, extends without substantial modification to the case of nonlinear phases satisfying the above hypothesis. The main difference is that the exact and approximate solutions are regarded as functions on $\Omega$. In the next section, nonlinear problems with nonlinear phases are considered, and the linear case can be extracted as a special case of those computations. In the absence of sources, that is when $b=0$, the principal profile is determined from the equations

$$
\pi(d \phi(x)) L_{1}(\partial) \pi(d \phi(x)) a_{0}=0, \quad \pi(d \phi(x)) a_{0}(x)=a_{0}(x) .
$$

If for all $x, \underline{\beta}:=d \phi(x)$ satisfies the constant multiplicity hypothesis of Proposition 3.2, then that result implies that the differential operator on the left is equal to

$$
\pi(d \phi(x)) A_{0} \pi(d \phi(x))\left(\frac{\partial}{\partial t}-\left.\sum_{j=0}^{d} \frac{\partial \tau(\eta)}{\partial \eta_{j}}\right|_{\eta=\partial_{y} \phi(x)} \frac{\partial}{\partial y_{j}}\right)+\pi(d \phi(x))\left(L\left(\partial_{x}\right) \pi(d \phi(x))\right) .
$$

The essential part is a variable coefficient vector field

$$
V\left(d \phi(x) ; \partial_{x}\right)=\partial_{t}+v_{g}(d \phi) . \partial_{x}
$$

which at each $x$ has speed given by the group velocity associated to $d \phi(x)$.

Equation (3.39) reads

$$
\left(\partial_{t}+v_{g}(d \phi) . \partial_{y}+\rho(x)\right)\left(\pi(d \phi) A_{0} \pi(d \phi) a_{0}\right)=0,
$$


where $\rho(x) \in \operatorname{Hom}(\operatorname{ker} L(i d \phi(x))$ is defined by

$$
\pi(d \phi)\left(\left(L\left(\partial_{x}\right) \pi(d \phi(x))-\left(V\left(d \phi ; \partial_{x}\right) \pi(d \phi) A_{0} \pi(d \phi)\right)\right) \pi(d \phi)=\rho(x) \pi(d \phi) A_{0} \pi(d \phi) .\right.
$$

If $\pi$ has rank one, then $\rho$ is scalar valued.

As in the case of nondispersive geometric optics the transport equation (3.41) yields an energy balance law in tubes of rays. The symmetric part of the operator $\rho$, which yields growth or decay in the transport, compensates the shrinking or expansion of ray tubes. The analysis is as in the nondispersive case presented, for example, in Ref. 25.

\section{APPROXIMATE SOLUTIONS OF SEMILINEAR DISPERSIVE SYSTEMS}

\section{A. Equations for the profiles}

The first two problems presented by nonlinearity are the creation and interaction of harmonics and the fact that the amplitude of the principal term becomes important. With the notation $\phi(x) \in C^{\infty}(\Omega)$ from the last subsection, we seek approximate solutions

$$
u^{\epsilon}=\epsilon^{p} a_{0}(x, \phi(x) / \epsilon)+\text { h.o.t. }
$$

where $a_{0}(x, \theta)$ is periodic in $\theta$. Fractional values of $p$ do occur. Taylor expansion of $F\left(u^{\epsilon}\right)$ introduces terms in $\epsilon^{m p}$ for all $m \in \mathbf{N}$.

From the last section we know that the equation of evolution for the coefficient of $\epsilon^{n}$ comes from the $\epsilon^{n+1}$ term in the expansion of $L u^{\epsilon}+F\left(u^{\epsilon}\right)$. This suggests that the set of exponents appearing in our ansatz be closed under multiplication by non-negative integers and by addition of 1. As we will see, the natural indices $p$ are rational, so that closure under multiplication by integers alone is sufficient.

Seek asymptotic solutions of the form

$$
u^{\epsilon}=\epsilon^{p} a(\epsilon, x, \phi(x) / \epsilon), \quad \text { where } \quad a(\epsilon, x, \theta) \sim \sum_{j \in p N} \epsilon^{j} a_{j}(x, \theta) .
$$

and $a(\epsilon, x, \theta)$ and $a_{j}(x, \theta)$ are smooth functions $2 \pi$ periodic with respect to $\theta$. For simplicity consider the differential equation without sources, that is $b=0$. This leads to the problem

$$
L\left(\epsilon \partial_{x}\right) u^{\epsilon}+F\left(u^{\epsilon}\right):=L_{1}\left(\epsilon \partial_{x}\right) u^{\epsilon}+L_{0} u^{\epsilon}+F\left(u^{\epsilon}\right) \sim 0 .
$$

Order $J$ hypothesis: The nonlinear term $F$, is a smooth function of its arguments whose partial derivatives of order less than or equal to $J-1 \geqslant 1$ vanish at the origin. Then the Taylor expansion at the origin is

$$
F(u)=\Phi(u)+O\left(|u|^{J+1}\right),
$$

where $\Phi$ is a homogeneous polynomial in $u, \bar{u}$ of degree $J \geqslant 2$.

In particular, $u=0$ satisfies the equation $0=L\left(\epsilon \partial_{x}\right) u+F(u)$. The function $u^{\epsilon}$ defined by (4.1) is a perturbation of this background state.

Examples: If $F$ is a homogeneous polynomial, then $F=\Phi$. For example, $F(u)=|u|^{2} u$ with $J=3$. The anharmonic oscillator model is an example with $J=3$. When the hypothesis is satisfied for $J \geqslant 3$, then it is satisfied for all $2 \leqslant J^{\prime}<J$.

The term $L(\epsilon \partial) u^{\epsilon}$ is of order $\epsilon^{p}$ while $F\left(u^{\epsilon}\right)$ is of order $\epsilon^{p J}$. The equation of evolution for $a_{0}$ comes from the $\epsilon^{p+1}$ term in $L u^{\epsilon}+F\left(u^{\epsilon}\right)$. We want this equation to involve $F$. This leads to the following choice of amplitude. 
Definition. For nonlinearities satisfying (4.3), the standard normalization is to choose $p$ so that $p J=p+1$, that is

$$
p:=\frac{1}{J-1}
$$

With this choice, $F\left(u^{\epsilon}\right)$ is smaller than the other two terms on the left of (4.2). For this reason the nonlinearity does not affect the definition of the dispersion relation. The nonlinearity is important at the next order which determines the evolution of the principal profile.

Examples: For quadratic nonlinearities, $p=1$, while for cubic nonlinearities, $p=\frac{1}{2}$. For the Klein-Gordon equation,

$$
\left(\epsilon^{2} \square+1\right) u+F\left(\epsilon \nabla_{x} u\right)=0,
$$

one has the same rules as for first order dispersive systems, that is for quadratic and cubic $F$, the standard normalizations are $p=1$ and $p=\frac{1}{2}$, respectively.

Compute

$$
L\left(\epsilon \partial_{x}\right) u^{\epsilon}=c(\epsilon \cdot x, \phi(x) / \epsilon),
$$

where

$$
c(\epsilon, x, \theta):=L_{1}\left(\epsilon \partial_{x}\right) a+L_{0} a+L_{1}(d \phi(x)) \partial_{\theta} a+F(a) .
$$

The strategy is to expand

$$
c(\epsilon, x, \theta) \sim \epsilon^{p} \sum_{j \in p \mathbf{N}} \epsilon^{j} c_{j}(x, \theta),
$$

and to choose the coefficients $a_{j}$ so that all the $c_{j}$ vanish identically.

Setting the coefficient $c_{0}$ of $\epsilon^{p}$ equal to zero yields

$$
0=L\left(d \phi(x) \partial_{\theta}\right) a_{0}=\left(L_{1}(d \phi(x)) \partial_{\theta}+L_{0}\right) a_{0} .
$$

As in the last section there is a hyperbolic-elliptic dichotomy in the analysis of this equation. Introduce the Fourier series

$$
a_{0}(x, \theta)=\sum_{m \in \mathbf{Z}} a_{0, m}(x) e^{i m \theta}
$$

to find

$$
0=L(\operatorname{imd} \phi) a_{0, m}:=\left(L_{1}(\operatorname{imd} \phi(x))+L_{0}\right) a_{0, m} .
$$

If all the matrices $L(i m d \phi)$ are nonsingular, the $a_{0, m}$ all vanish. On the other hand, if there is an $m \neq 0$ such $L(\operatorname{imd} \phi(x))$ is singular for all $x$, the corresponding terms lead to propagating oscillations.

There are intermediate cases where $L(\operatorname{imd} \phi(x))$ may be singular at some but not all $x$. The crucial coherence hypothesis is imposed to avoid these.

Coherence hypothesis: For each $m \in \mathbf{Z} \backslash \mathbf{O}$, if $L(i m d \phi(x))$ is singular for one $x \in \Omega$, then it is singular for all $x \in \Omega$. When $L(\operatorname{imd} \phi(x))$ is singular, the matrix $\pi(m d \phi(x))$ is assumed to be a smooth function of $x$. 
The simplest example is when $\phi(x)$ is a linear function of $x$, in which case $L(\operatorname{imd} \phi(x))$ does not depend on $x$. The coherence hypothesis says that for the harmonics $m \phi$, the eikonal equation is either satisfied for all $x$ or for none.

For nondispersive geometric optics, that is problems with $L(\partial)$ rather than $L(\epsilon \partial)$, the eikonal equation is homogeneous so that $m \phi$ is automatically a solution of the eikonal equation for all $m$. Thus the analogue of the above hypothesis for single phase nondispersive geometric optics is automatically satisfied. Coherence hypotheses play a crucial role in nondispersive multiphase nonlinear geometric.

The analysis is simplified if there are only a finite number of $m$ for which $m d \phi$ satisfies the eikonal equation. This is guaranteed by the following hypothesis which is satisfied in all the physical models we have studied.

Strong finiteness hypothesis: $L_{1}(i d \phi(x))$ is nonsingular for all $x \in \Omega$.

Writing

$$
L(\operatorname{imd} \phi(x))=m\left(L_{1}(i d \phi(x))+\frac{1}{m} L_{0}\right)
$$

shows that there is an $m_{0} \geqslant 0$ so that $L(\operatorname{imd} \phi(x))^{-1}$ exists for $|m| \geqslant m_{0}$ and that uniformly on compact subsets of $\Omega$,

$$
\left\|L(\operatorname{imd} \phi(x))^{-1}\right\|=O\left(\frac{1}{m}\right)
$$

Let $\mathscr{l} b$ denote the finite set

$$
\mathscr{C l}:=\{m \in \mathbf{Z}: \operatorname{det} L(\text { imd } \phi)=0\} .
$$

Example: If $\phi$ satisfies the eikonal equation (3.19) for the Klein-Gordon equation, then for $m \phi$, compute

$$
(m \phi)_{t}^{2}-\left|(m \phi)_{y}\right|^{2}+1=\left(m^{2}-1\right)\left(\phi_{t}^{2}-\left|\phi_{y}\right|^{2}\right)=1-m^{2} .
$$

Thus for $m \neq \pm 1, m \phi$ does not satisfy the eikonal equation. In addition, with $L_{2}$ denoting the part homogeneous of degree 2 ,

$$
L_{2}(d \phi)=\phi_{t}^{2}-\left|\phi_{y}\right|^{2}=-1
$$

which vanishes nowhere so the strong finiteness hypothesis is satisfied.

Example: Suppose that $\phi$ satisfies the eikonal equation (3.37) for the Lorentz model. A calculation like that in Sec. III shows that

$$
\operatorname{det} L_{1}(\tau, \eta)=\tau^{8}\left(\tau^{2}-|\eta|^{2}\right)^{2}
$$

It follows that for such eikonal $\phi$.

$$
\operatorname{det} L_{1}(d \phi)=\frac{-\gamma \phi_{t}^{8}}{\phi_{t}^{2}-1}
$$

Thus if $\phi_{t} \notin\{0,1\}$, this is nonsingular. In addition, $m \phi$ satisfies

$$
\left(\left(m \phi_{t}\right)^{2}-1\right)\left(\left(m \phi_{t}\right)^{2}-\left|m \phi_{y}\right|^{2}\right)+\gamma\left(m \phi_{t}\right)^{2}=\left(m^{2}-1\right)\left(\left(m \phi_{t}\right)^{2}-\left|m \phi_{y}\right|^{2}\right)=\frac{\left(m^{2}-1\right) \gamma\left(m \phi_{t}\right)^{2}}{\phi_{t}^{2}-1} .
$$


So (3.38) shows that if $\phi_{t} \notin\{0,1,1+\gamma\}$, then $m \phi$ is eikonal only for $m \in\{0,1,-1\}$.

It is rare that nontrivial harmonics satisfy the eikonal equation. In the scientific literature, when $m \phi$ is eikonal the phases $\phi$ and $m \phi$ are often said to be phase matched.

Aside: The possibility of an infinity of harmonics which satisfy the eikonal equation can be handled with a suitable small divisor hypothesis. What is needed is that there is a constant $C$ and an integer $M$ such that

$$
\left\|\left(L_{1}(i m d \phi(x))+L_{0}\right)^{-1}\right\| \leqslant C(1+|m|)^{M},
$$

for $m d \phi(x) \notin \epsilon$ char and also

$$
\|Q(m d \phi(x))\| \leqslant C(1+|m|)^{M} .
$$

We do not pursue this point of view but impose the finiteness hypothesis above.

In order to treat the dichotomy of propagating versus nonpropagating oscillations, introduce the projection of the Fourier series on the set of harmonics which satisfy the eikonal equation.

Definition: When $d(x, \theta)$ is a $2 \pi$ periodic function in $\theta, \Pi \mathbf{\Pi} d$, the projection on $\epsilon$ char harmonics, is defined by

$$
\boldsymbol{\Pi} d:=\sum_{m \in \mathbf{Z}} \pi(m d \phi(x)) d_{m}(x) e^{i m \theta} .
$$

In addition, $\mathbf{Q}$ denotes the partial inverse of $L\left(d \phi \partial_{\theta}\right)$ defined by

$$
\mathbf{Q} d:=\sum_{m \in \mathbf{Z}} Q(m d \phi(x)) d_{m}(x) e^{i m \theta} .
$$

Remarks: (1) In the definition of $\boldsymbol{\Pi}$ one could have taken the sum only over $m \in \mathscr{L} b$ since for the other $m, \pi(m d \phi(x))=0$.

(2) The finiteness assumption shows that $\Pi d$ is a trigonometric polynomial. That is, it has at most a finite number of nonvanishing Fourier coefficients each of which is a function of $x$. Thus, $\boldsymbol{\Pi} d$ is determined by a finite number, of functions of $x$. It is useful to think of the image of $\boldsymbol{\Pi}$ as consisting of vector valued functions of $x$.

(3) The estimate for $L(\text { imd } \phi)^{-1}$ following the strong finiteness hypothesis shows that $\mathbf{Q}$ is a continuous map of $C^{\infty}\left(\Omega \times S^{1}\right)$ to itself. $\mathbf{Q}$ is an operator of order -1 in $\partial_{\theta}$.

With this definition, Eq. (4.6) is equivalent to

$$
\Pi a_{0}=a_{0} .
$$

We next find evolution equations which determine $\Pi a_{0}$ from its initial data. Setting $c_{1}=0$ in the expansion of $c(\epsilon, x, \theta)$ yields

$$
L\left(d \phi(x) \partial_{\theta}\right) a_{1}+L_{1}\left(\partial_{x}\right) a_{0}+\Phi\left(a_{0}\right)=0 .
$$

Multiplying by $\Pi$, equivalently setting $\Pi c_{1}=0$ eliminates the $a_{1}$ term to yield

$$
\Pi L_{1}\left(\partial_{x}\right) a_{0}+\Pi \Phi\left(a_{0}\right)=0 .
$$

Using (4.9) yields

$$
\boldsymbol{\Pi} L_{1}\left(\partial_{x}\right) \boldsymbol{\Pi} a_{0}+\boldsymbol{\Pi} \Phi\left(\boldsymbol{\Pi} a_{0}\right)=0 .
$$


The examples in the next subsection and the existence theorem in Sec. IV C show that the nonlinear evolution equation (4.11) determines the finite-dimensional vector-valued function $a_{0}=\boldsymbol{\Pi} a_{0}$ from its initial values, at least locally in time.

Next a linear recurrence to determine $a_{j}$ for $j>0$ is found. For $j>0$, the coefficient $c_{j}$ of $\epsilon^{p+j}$ is given by

$$
c_{j}=L\left(d \phi(x) \partial_{\theta}\right) a_{j}+L_{1}\left(\partial_{x}\right) a_{j-1}+\Phi^{\prime}\left(a_{0}\right) a_{j-1}+G_{j}\left(a_{l<j-1}\right)
$$

where by convention we set $a_{k}=0$ when $k<0$.

Once the $a_{l}$ are known for $l<j,(I-\boldsymbol{\Pi}) a_{j}$ is determined by setting $\mathbf{Q} c_{j}=0$ to find

$$
(I-\boldsymbol{\Pi}) a_{j}=-\mathbf{Q}\left(L_{1}\left(\partial_{x}\right) a_{j-1}+\Phi^{\prime}\left(a_{0}\right) a_{j-1}+G_{j}\left(a_{l<j-1}\right)\right)
$$

Recall that $j=n p$ and $p$ may be smaller than one. An interesting special case is the range $0 \leqslant j<1$ where the source terms with $l \leqslant j-1$ all vanish to give

$$
\Pi a_{j}=a_{j} \text { for } \quad 0 \leqslant j<1 \text {. }
$$

The equation $\Pi c_{j+1}=0$ is used to determine the propagating part $\Pi a_{j}$ of $a_{j}$. One has

$$
\boldsymbol{\Pi} L_{1}\left(\partial_{x}\right) \Pi a_{j}+\Pi \Phi^{\prime}\left(a_{0}\right) a_{j}=-\Pi\left(G_{j}\left(a_{l<j}\right)+L_{1}\left(\partial_{x}\right)(I-\Pi) a_{j}\right)
$$

where the right-hand side is known from (4.13) and the inductive hypothesis. For $j \geqslant 1$, this yields a finite set of coupled linear hyperbolic equations which determine $\mathbf{\Pi} a_{j}$ from their initial data.

Example: In the range $0<j<1$, the equation is the linear equation

$$
\boldsymbol{\Pi} L_{1}\left(\partial_{x}\right) \Pi a_{j}+\Pi \Phi^{\prime}\left(a_{0}\right) a_{j-1}=0 \quad \text { for } \quad 0<j<1 .
$$

Thus if the initial values of these profiles vanish, the profiles vanish for all time. In this case the first profile after the principal profile $a_{0}$ is the profile $a_{1}$. This yields an interesting class of expansions of type (4.1) where the elements of $p \mathbf{N}$ between 0 and 1 are absent.

Theorem 4.1: Suppose that $\Omega \subset \mathbf{R}^{1+d}$ is an open set and the phase $\phi \in C^{\infty}(\Omega ; \mathbf{R})$ satisfies the coherence and strong finiteness hypotheses, and that

$$
u^{\epsilon}=\epsilon^{p} a(\epsilon, x, \phi(x) / \epsilon) \quad \text { with } \quad a(\epsilon, x, \theta) \sim \sum_{j \in p \mathbf{N}} \epsilon^{j} a_{j}(x, \theta)
$$

in $C^{\infty}\left(\Omega \times S^{1}\right)$. Then

$$
L(\epsilon \partial) u^{\epsilon}+F\left(u^{\epsilon}\right) \sim 0 \quad \text { in } \quad C^{\infty}(\Omega)
$$

if (4.7), (4.9), (4.11), and the infinite sequence of relations (4.13) and (4.14) are satisfied.

\section{B. Examples}

\section{The nonlinear Klein-Gordon equation}

The equation is

$$
\epsilon^{2} \square u+u+F\left(\epsilon \nabla_{x} u\right)=0,
$$

where the Taylor expansion of $F=\Phi(u)+$ h.o.t begins with nonzero terms homogeneous of degree $J$. The ansatz for the real scalar-valued $u^{\epsilon}$ is given by (4.1) with $p=1 /(J-1)$. One constructs solutions with residual $O\left(\epsilon^{\infty}\right)$. The term of order $\epsilon^{p}$ yields the eikonal equation (3.36). As already verified the strong finiteness and coherence hypotheses are automatic and the only eikonal harmonics are those with $m= \pm 1$. Reality implies that $a_{0,1}(x)=\bar{a}_{0,-1}(x):=a(x)$ and then 


$$
u^{\epsilon} \sim \epsilon^{p} a_{0}(x, \phi(x) / \epsilon)+\text { h.o.t., } \quad \text { with } a_{0}(x, \theta)=a(x) e^{i \theta}+\bar{a}(x) e^{-i \theta} .
$$

The projection $\boldsymbol{\Pi}$ selects the Fourier components \pm 1 that is

$$
\Pi \sum d_{m}(x) e^{i m \theta}=d_{1}(x) e^{i \theta}+d_{-1}(x) e^{-i \theta} .
$$

The analogue of Eq. (4.11) shows that the principal amplitude is determined from its initial data by the transport equation

$$
2\left(\phi_{t} \partial_{t}-\partial_{y} \phi, \nabla_{y}+\frac{\square \phi}{2}\right) \partial_{\theta} a_{0}+\Phi\left(d \phi(x) \partial_{\theta} a_{0}(x, \theta)\right)=0 .
$$

The nonlinear term is equal to

$$
\Phi\left(d \phi \partial_{\theta} a_{0}\right)=\Phi(d \phi) \Pi\left(\left(i a e^{i \theta}-i \bar{a} e^{-i \theta}\right)^{J}\right) .
$$

Even $J$ : If $J$ is even, there are no terms in $e^{ \pm i \theta}$ so the projection is equal to zero. In this case the transport equation is linear. The nonlinear term does not affect the leading term in the asymptotic expansion.

Odd $J:$ If $J=2 m+1$ is odd, then one generates an $e^{i \theta}$ term on choosing $m+1$ factors $i a e^{i \theta}$ and $m$ factors $-i \bar{a} e^{-\theta}$. Reasoning similarly for the coefficient of $e^{-i \theta}$ shows that

$$
\boldsymbol{\Pi}\left(\left(i a e^{i \theta}-i \bar{a} e^{-i \theta}\right)^{J}\right)=\frac{J !}{m !(m+1) !}\left(i a|a|^{J-1} e^{i \theta}-i \vec{a}|a|^{J-1} e^{-i \theta}\right) .
$$

Thus the transport equation for $a_{0}$ is equivalent to the transport equation

$$
2\left(\phi_{t} \partial_{t}-\partial_{y} \phi \cdot \nabla_{y}+\frac{\square \phi}{2}\right) a+\frac{J ! \Phi(d \phi(x))}{(m !)(m+1) !} a|a|^{J-1}=0 .
$$

This is an explicitly solvable nonlinear ordinary differential equation along the integral curves of the vector field appearing on the left. As a special case consider $F(\nabla u)=u_{t}^{3}$ and linear phase $\phi=\tau t+y \cdot \eta$ satisfying the eikonal equation $\tau^{2}=|\eta|^{2}+1$. The equation for $a$ is the dissipative cubic transport

$$
\left(\tau \partial_{t}-\eta . \partial_{y}\right) a+\frac{3 \tau^{3}}{2} a|a|^{2}=0
$$

A second example is the phase $\phi(t, y):=\sqrt{2} t+|y|-1$ which satisfies the eikonal equation away from $y=0$. The group velocities are equal to $1 / \sqrt{2}<1$. If initial data for the amplitudes $a_{j}$ vanish on $|y| \leqslant 1$, then, for the dissipative nonlinearity $u_{t}^{3}$, the transport equations for the $a_{j}$ are solvable for $0 \leqslant t<\sqrt{2}$. In this way one can construct infinitely accurate approximate solutions in $\left[0, \sqrt{2}\left[\times \mathbf{R}^{d}\right.\right.$ which correspond to waves surrounding and approaching the origin, a point where the phase is not defined.

\section{Small amplitudes}

If the system (4.2) has nonlinearity of order $J \geqslant 3$ in the sense of (4.3) and $2 \leqslant J^{\prime}<J$, then the hypotheses are satisfied for $J^{\prime}$ and one can construct approximate solutions with the standard normalization for $J^{\prime}$, that is, of the form $(4.1)$ for $p^{\prime}:=1 /\left(J^{\prime}-1\right)>p$. These solutions are smaller in amplitude than the standard scaling for $J$ and the equation defining the principal profile $a_{0}$ is linear. 


\section{The anharmonic oscillator model}

Suppose that $\tau \notin\{0, \pm 1\}$ satisfies the $\epsilon$-characteristic equation (3.13). Equations (3.14) show that the orthogonal projector onto the kernel of $L(i \tau, i \eta)$ satisfies for $\mathbf{v} \perp \eta$,

$$
\pi(\tau, \eta)(0,0,0, \mathbf{v})=\frac{i \tau}{1-\tau^{2}}\left(1+\frac{\eta^{2}}{\tau^{2}}+\frac{\gamma^{2}\left(1+\tau^{2}\right)}{\left(1-\tau^{2}\right)^{2}}\right)^{-1}\left(\mathbf{v}, \frac{-\eta \wedge \mathbf{v}}{\tau}, \frac{\gamma \mathbf{v}}{1-\tau^{2}}, \frac{i \tau \gamma}{1-\tau^{2}} \mathbf{v}\right)
$$

If $\phi$ satisfies the eikonal equation (3.37), then $m \phi$ is eikonal only for $m \in\{0, \pm 1\}$ and the coherence and strong finiteness hypotheses are satisfied. The operator $\boldsymbol{\Pi}$ is then defined using three spectral projectors, those on $\operatorname{ker} L( \pm d \phi)$ and $\operatorname{ker} L_{0}$. The first two kernels are described by (4.21) with $(\tau, \eta)=d \phi$. $\operatorname{ker} L_{0}$ is the set of vectors satisfying $Q=P-\gamma E=0$, so $\operatorname{ker} L_{0}=\{(E, B, \gamma E, 0)\}$ and

$$
\pi(0)(E, B, P, Q)=(0, B, 0,0)+\frac{1}{1+\gamma^{2}}\left(E+\gamma P, 0, \gamma E+\gamma^{2} P, 0\right) .
$$

The principal profile has the form

$$
a_{0}(x, \theta)=a_{+}(x) e^{i \theta}+a_{0}(x)+a_{-}(x) e^{-i \theta},
$$

where the polarization (4.8) holds exactly when

$$
\pi( \pm d \phi(x)) a_{ \pm}(x)=a_{ \pm} \quad \text { and } \quad \pi(0) a_{0}(x)=a_{0}(x) .
$$

Reality requires

$$
a_{\mp}=\bar{a}_{ \pm} \quad \text { and } \quad a_{0}=\overline{a_{0}} .
$$

Introduce components

$$
a_{ \pm}:=\left(E_{ \pm}, B_{ \pm}, P_{ \pm}, Q_{ \pm}\right), \quad a_{0}:=\left(E_{0}, B_{0}, P_{0}=\gamma E_{0}, Q_{0}=0\right) .
$$

The evolution equation for the profile involves

$$
\Phi(u)=\left(0,0,0, \alpha|P|^{2} P\right) .
$$

The first important observation is that $\pi(0) \Phi(u)=0$, so the equation of evolution for $a_{0}$ is

$$
\pi(0) L_{1}(\partial) a_{0}(x)=0 .
$$

Note in particular that $a_{0}=0$ is a solution. This special case is examined in more detail below.

Equation (4.22) shows that Eq. (4.28) holds if and only if the second component and the sum of the first plus $\gamma$ times the third component of

$$
L_{1}(\partial) A_{0}=\left(\partial_{t} E_{0}-\operatorname{curl} B_{0}, \partial_{t} B_{0}+\operatorname{curl} E_{0}, P_{0}, Q_{0}\right)
$$

vanish. Using (4.22) and the relation $P_{0}=\gamma E_{0}$ from (4.24) shows that when (4.24) holds, (4.28) is equivalent to the modified Maxwell equations

$$
\partial_{t} B_{0}+\operatorname{curl} E_{0}=0, \quad\left(1+\gamma^{2}\right) \partial_{t} E_{0}-\operatorname{curl} B_{0}=0 .
$$

Turn next to the determination of $a_{ \pm}$. Once $a_{0}$ is determined, the amplitude $a_{+}$is determined from the evolution equation 


$$
\left(\partial_{t}+v_{g} . \partial_{y}+\rho(x)\right)\left(\pi_{+} A_{0} a_{+}\right)+\pi_{+} \Phi\left(a_{+} e^{i \theta}+a_{0}+\bar{a}_{+} e^{-i \theta}\right)=0 .
$$

The nonlinear term from (4.27) is $\alpha$ times

$$
|P|^{2} P=P . \bar{P} P=\left(P_{+} e^{i \theta}+P_{0}+\bar{P}_{+} e^{-i \theta}\right) \cdot\left(P_{+} e^{i \theta}+P_{0}+\bar{P}_{+} e^{-i \theta}\right)\left(P_{+} e^{i \theta}+P_{0}+\bar{P}_{+} e^{-i \theta}\right),
$$

where the dot product is that without complex conjugates. The operator $\pi_{+}$picks out the terms in $e^{i \theta}$ and multiplies them by $\pi(\tau, \eta)$, with $(\tau, \eta)=d \phi$. There are six terms with the right exponent. Three of them have two factors $e^{i \theta}$ and one factor $e^{-i \theta}$, while the other three have two $P_{0}$ 's and one $e^{i \theta}$.

For simplicity we treat only the case $a_{0}=0$. The terms in $e^{i \theta}$ are then

$$
2 P_{+} \cdot \bar{P}_{+} P_{+}+P_{+} \cdot P_{+} \bar{P}_{+}=2\left|P_{+}\right|^{2} P_{+}+P_{+} \cdot P_{+} \bar{P}_{+} .
$$

The polarization from (4.20) and (4.24) shows that

$$
P_{+}=\frac{\gamma}{1-\left(\partial_{t} \phi\right)^{2}} E_{+}
$$

Thus,

$$
\pi_{+} \Phi(a)=\alpha\left(\frac{\gamma}{1-\left(\partial_{t} \phi\right)^{2}}\right)^{3} \pi_{+}(d \phi)\left(0,0,0,2\left|E_{+}\right|^{2} E_{+}+E_{+} \cdot E_{+} \bar{E}_{+}\right) .
$$

Equation (4.21) can be used to compute the action of the projection $\pi(d \phi)$. Equating the $E$ components on the two sides of (4.30) and using the formula before Proposition 3.4 yields the cubic transport equation

$$
0=\frac{1}{i \alpha}\left(\partial_{t}+v_{g} . \partial_{y}+\rho(x)\right)\left(\mu(d \phi) E_{+}\right)+c(d \phi)\left(2\left|E_{+}\right|^{2} E_{+}+\left(E_{+} \cdot E_{+}\right) \bar{E}_{+}\right),
$$

where

$$
c(d \phi):=\left(\frac{\gamma}{1-\left(\partial_{t} \phi\right)^{2}}\right)^{3}\left(\frac{\partial_{t} \phi}{1-\left(\partial_{t} \phi\right)^{2}}\right)\left(1+\frac{\left|\partial_{y} \phi\right|^{2}}{\left(\partial_{t} \phi\right)^{2}}+\frac{\gamma^{2}\left(\left(1+\partial_{t} \phi\right)^{2}\right)}{\left(1-\left(\partial_{t} \phi\right)^{2}\right)^{2}}\right)^{-1} .
$$

Note that the denominators of $\mu(d \phi)$ and $c(d \phi)$ are identical, which yields a significant simplification for linear phases. The other components of $a_{+}$can be found from $E_{+}$using the polarization (4.20). The cubic ordinary differential equation (4.32) is explicitly solvable (see Ref. 27, pp. 51-53). Taking the imaginary part of the scalar product with $\bar{E}_{+}$yields

$$
\left(\partial_{t}+v_{g} . \partial_{y}+\operatorname{Re} \rho(x)\right)\left(\mu(d \phi)\left|E_{+}\right|^{2}\right)=0,
$$

which is the natural conservation of energy.

\section{Solvability of the profile equations}

In all the examples of the previous sections, the equations determining the profiles, though not exactly easy to find, were easy to solve once found. Even more, the nonlinear evolution equations for the principal profiles were globally solvable when the phases were linear. With this experience, the next result guaranteeing local existence and uniqueness of the infinite family of profiles should not be surprising.

A key ingredient is solving initial value problems for $\Pi L_{1}\left(\partial_{x}\right) \Pi$. The operator $\Pi L_{1}\left(\partial_{x}\right) \Pi$ maps trigonometric polynomials 


$$
\sum_{m \in \mathscr{l} b} a_{m}(x) e^{i m \theta}, \quad \pi(m d \phi) a_{m}=a_{m}
$$

to themselves. To such a polynomial associate the finite set of coefficient functions $a_{m}$ with $m \in \mathscr{L l}$. Then $\Pi L_{1}\left(\partial_{x}\right) \Pi$ acts as

$$
\operatorname{diag}\left\{\pi(m d \phi) L_{1}(\partial) \pi(m d \phi): m \in \mathscr{C}\right\}
$$

The next hypothesis is crucial for the solvability of initial value problems.

Determinacy hypothesis: $T_{1}>0$ and $\Omega \cap\left(\left[0, T_{1}\right] \times \mathbf{R}^{d}\right)$ is a domain of determinacy for each of the symmetric hyperbolic operators $\pi(m d \phi) L_{1}\left(\partial_{x}\right) \pi(m d \phi), m \in \mathscr{l l}$.

Example: It is often the case that each of the operators $\pi(m d \phi) L_{1}\left(\partial_{x}\right) \pi(m d \phi)$ is a transport operator in which case the hypothesis asserts that every backward group velocity ray from a point in $\Omega \cap\left(\left[0, T_{1}\right] \times \mathbf{R}^{d}\right)$ reaches $t=0$ without leaving $\Omega \cap\left(\left[0, T_{1}\right] \times \mathbf{R}^{d}\right)$.

Theorem 4.2: Suppose that the phase $\phi \in C^{\infty}(\Omega)$ and $T_{1}>0$ satisfy the coherence, strong finiteness, and determinacy hypothesis. In addition, for $0 \leqslant j \in p \mathbf{N}$ suppose given

$$
g_{j}(y, \theta) \in C_{0}^{\infty}\left(\mathbf{R}^{d} \times S^{1}\right), \quad \operatorname{supp} g \subset(\Omega \cap\{t=0\}) \times S^{1} \quad \text { with } \quad \Pi g_{j}=g_{j} .
$$

Then there is a $\left.T \in] 0, T_{1}\right]$ and a unique solution $a_{0} \in C^{\infty}\left((\Omega \cap\{0 \leqslant t \leqslant T\}) \times S^{1}\right)$ of (4.8) and (4.11) such that $\boldsymbol{\Pi} a_{0}(0, y)=g_{0}(y)$.

Moreover for $j>0$, the infinite sequence of equations (4.13) and (4.14) uniquely determine functions $a_{j} \in C^{\infty}\left((\Omega \cap\{0 \leqslant t \leqslant T\}) \times S^{1}\right)$ satisfying the initial condition $\Pi a_{j}(0, y)=g_{j}$.

Proof: The polarization (4.9) implies that

$$
a_{0}=\Pi a_{0}=\sum_{m \in \mathscr{b}} a_{m}(x) e^{i m \theta}, \quad \pi(m d \phi(x)) a_{m}=a_{m} .
$$

The unknown is the finite set of functions $a_{m}(x), m \in \mathscr{C}$, satisfying $\pi(m d \phi(x)) a_{m}=a_{m}$. Thus $a_{0}$ is a section of a finite-dimensional vector subbundle of $\oplus_{m \in \mathscr{l}} \mathbf{C}^{N}$. The fiber changes from point to point because of the projection $\pi(m d \phi(x))$.

In Eq. (4.11) expand

$$
\Pi \Phi\left(\sum_{m \in \mathscr{M}} a_{m}(x) e^{i m \phi(x)}\right)=\sum_{m \in \mathscr{C}} c_{m}\left(x,\left\{a_{\mu}(x)\right\}\right) e^{i m \phi(x)},
$$

where the value of $c_{m}$ at $x$ is a polynomial of order $J$ in the values $\left\{a_{\mu}(x)\right\}$. The $c_{m}$ are smooth in $x$ and satisfy

$$
\pi(m d \phi(x)) c_{m}(x, .)=c_{m}(x, .) .
$$

The equations for $a_{0}$ takes the form of \#/b coupled equations

$$
\pi(m d \phi) L_{1}\left(\partial_{x}\right) \pi(m d \phi) a_{m}+c_{m}\left(x,\left\{a_{\mu}(x)\right\}\right)=0 .
$$

Proposition 3.2 shows that (4.35) is a semilinear symmetric hyperbolic system for the functions $a_{m}$ which take values in $\operatorname{ker} L(i m d \phi(x))$. Thanks to the domain of determinacy hypothesis, local existence and uniqueness is a consequence of classical results for semilinear hyperbolic systems.

The recurrence for the succeeding profiles leads to algebraic equations for $(I-\Pi) a_{j}$ and linear hyperbolic systems, with the same principal part as in (4.35), for $\Pi a_{j}$. The sources in those systems are given in terms of already determined quantities. Again existence and uniqueness are classical. 
Remark: This existence theorem is simpler than those required for nondispersive systems where the unknowns are functions of $x$ and one or more auxiliary variables. In the present case the dependence on the auxiliary variable $\theta$ simplifies thanks to the strong finiteness assumption.

\section{APPROXIMATE SOLUTIONS OF QUASILINEAR DISPERSIVE SYSTEMS}

Suppose that

$$
L(u, \partial):=\sum_{j=0}^{d} A_{\mu}(u) \frac{\partial}{\partial x_{\mu}}
$$

is a quasilinear symmetric hyperbolic operator near $u=0$ in the sense that the coefficients $A_{\mu}$ are smooth Hermitian symmetric functions of their arguments on a neighborhood of 0 . The vectorvalued unknown may be either real or complex valued $u$. Correspondingly the coefficients need to be defined on neighborhoods in $\mathbf{R}^{N}$ or $\mathbf{C}^{N}$.

Seek approximate solutions $u^{\epsilon}$ to the quasilinear dispersive hyperbolic problem

$$
L\left(u^{\epsilon}, \epsilon \partial\right) u^{\epsilon}+L_{0} u^{\epsilon}+F\left(u^{\epsilon}\right)=0 .
$$

The approximate solutions have the form

$$
u^{\epsilon}(x)=\epsilon^{p} a(\epsilon, x, \phi(x) / \epsilon) \quad \text { with } \quad a(\epsilon, x, \theta)
$$

smooth and periodic in $\theta$.

Order J hypothesis: The nonlinear functions $F$ and $A_{\mu}$ are smooth on a neighborhood of 0 , and the nonlinear terms are of order $J \geqslant 2$ in the sense that

$$
|\alpha| \leqslant J-2 \Rightarrow \partial_{u, \bar{u}}^{\alpha} L(0, d \phi(x))=0 \quad \text { and } \quad|\beta| \leqslant J-1 \Rightarrow \partial_{u, \bar{u}}^{\beta} F(0)=0
$$

The Taylor polynomial of order $J$ of $F$ at the origin is denoted $\Phi(u)$ as in (4.3), while for $L$ we write

$$
A_{\mu}(u)-A_{\mu}(0)=\Lambda_{\mu}(u)+O\left(|u|^{J}\right), \quad \Lambda(u, \xi):=\sum_{\mu=0}^{d} \Lambda_{\mu}(u) \xi_{\mu}
$$

where the $\Lambda_{\mu}$ are polynomials homogeneous of degree $J-1$.

The reason for the discrepancy in the orders is because the coefficients $A$ appear in expressions $A(u) \epsilon \partial u$ so the order of the nonlinearity is one higher than the order of the zero of $A$.

The critical exponent is $p=1 /(J-1)$, in which case the nonlinear terms from $F$ and from $L(u, \epsilon \partial) u$ are both $O\left(\epsilon^{p+1}\right)$. As in Sec. IV, the ansatz (4.1) is in powers $\epsilon^{j}$ with $j \in p \mathbf{N}$.

Hypothesis: Suppose that $\phi \in C^{\infty}(\Omega ; \mathbf{R})$ satisfies the eikonal equation with respect to the linear dispersive operator $L(0, \epsilon \partial)$, and that the coherence, strong finiteness, and domain of dependence hypotheses are satisfied. The projector $\mathbf{\Pi}$ and partial inverse $\mathbf{Q}$ are defined as in Secs. III and IV, using the linear $\epsilon$-differential operator, $L(0, \epsilon \partial)$.

Example: The instantaneous nonlinear polarization from Sec. II C is an example with $J=3$.

Direct computation shows that

$$
L\left(u^{\epsilon}, \epsilon \partial\right) u^{\epsilon}+L_{0} u^{\epsilon}+F\left(u^{\epsilon}\right)=c(\epsilon, x, x d \phi(x) / \epsilon), \quad c(\epsilon, x, \theta) \sim \epsilon^{p} \sum_{j \in p \mathbf{N}} \epsilon^{j} c_{j}(x, \theta)
$$

The strategy is to choose the $a_{j}$ so that $c_{j}=0$ for all $j$. For $j<0$, define $a_{j}:=0$. Setting the coefficient $c_{j}=0$ for $0 \leqslant j<1$ yields 


$$
0=c_{j}=L\left(0, d \phi(x) \partial_{\theta}\right) a_{j}
$$

This is equivalent to

$$
\Pi a_{j}=a_{j} \quad \text { for } \quad 0 \leqslant j<1 .
$$

The main change with respect to the semilinear case occurs in the coefficient $c_{1}$. The new $O\left(\epsilon^{p+1}\right)$ quasilinear term is

$$
\sum_{\mu=0}^{d} \Lambda_{\mu}\left(a_{0}\right) \frac{\partial \phi}{\partial x_{\mu}} \partial_{\theta} a_{0}=\Lambda\left(a_{0}, d \phi\right) \partial_{\theta} a_{0} .
$$

With this notation, setting $c_{1}$ equal to zero yields

$$
0=c_{1}=L\left(0, d \phi \partial_{\theta}\right) a_{1}+L_{1}(0, \partial) a_{0}+\Lambda\left(a_{0}, d \phi\right) \partial_{\theta} a_{0}+g F\left(a_{0}\right) .
$$

Multiplying by $\Pi$ eliminates the $a_{1}$ term and yields the evolution equation for $\Pi a_{0}$

$$
0=\boldsymbol{\Pi} L_{1}(0, \partial) \Pi a_{0}+\Lambda\left(a_{0}, d \phi\right) \partial_{\theta} a_{0}+\Phi\left(a_{0}\right) .
$$

Here there is an important remark. Equation (5.8) looks like it is a quasilinear differential equation in $(x, \theta)$ because $\Lambda$ is homogeneous of degree $J-1$ in $a_{0}$. It would be quasilinear in the nondispersive case. ${ }^{10-13}$ However, with the finite set $\mathscr{L} 6$ one has

$$
a_{0}(x, \theta)=\sum_{m \in \mathscr{l}} a_{m}(x) e^{i m \theta} .
$$

As in Sec. IV C, binomial-type expansion defines interaction coefficients $c_{m}\left(x,\left\{a_{\mu}(x)\right\}\right)$ by

$$
\Pi\left(\Lambda\left(a_{0}, d \phi\right) \partial_{\theta} a_{0}+\Phi\left(a_{0}\right)\right)=\sum_{m \in \mathscr{l l}} c_{m}\left(x,\left\{a_{\mu}(x)\right\}\right) e^{i m \phi / \epsilon}, \quad \pi(\mu d \phi) c_{m}=c_{m} .
$$

Here $c_{\mu}$ is a polynomial of degree $J$ in $\left\{a_{m}(x)\right\}$ with coefficients depending smoothly on $x$. With the above notation, Eq. (5.8) becomes

$$
\pi(m d \phi(x)) L_{1}\left(0, \partial_{x}\right) \pi(m d \phi(x)) a_{m}+c_{m}\left(x,\left\{a_{\mu}(x)\right\}\right)=0 .
$$

This is identical in form to (4.35). Only the interaction coefficients $c_{m}$ have changed. The seemingly quasilinear term contributes semilinear terms like those from $F$.

Multiplying Eq. (5.7) by $\mathbf{Q}$ yields an expression for $(I-\boldsymbol{\Pi}) a_{1}$ in terms of $a_{0}$.

For $j \geqslant 2$, setting the coefficients $c_{j}$ equal to zero yields equations of the form

$$
L\left(0, d \phi \partial_{\theta}\right) a_{j}+\Phi^{\prime}\left(a_{0}\right) a_{j-1}+\Lambda\left(a_{0}, d \phi\right) \partial_{\theta} a_{j-1}+\Lambda_{u}^{\prime}\left(a_{0}, d \phi\right)\left(a_{j-1}\right) \partial_{\theta} a_{0}=G_{j}\left(a_{k<j-1}\right) .
$$

The expression $G$ involves derivatives of the functions $a_{m}$ even though that is not explicitly indicated.

Multiplying (5.12) by $\mathbf{Q}$ yields

$$
(I-\Pi) a_{j}=G_{j}\left(a_{k \leqslant j-1}\right) .
$$

This done, multiplying the case $j+1$ of (5.12) by $\Pi$ I yields a linear evolution equation 


$$
\boldsymbol{\Pi} L_{1}(0, \partial) \Pi a_{j}+\Pi\left\{\Phi^{\prime}\left(a_{0}\right) a_{j-1}+\Lambda\left(a_{0}, d \phi\right) \partial_{\theta} a_{j-1}+\Lambda_{u}^{\prime}\left(a_{0}, d \phi\right)\left(a_{j-1}\right) \partial_{\theta} a_{0}\right\}=\Pi G_{j}\left(a_{k<j-1}\right),
$$

which determines $\Pi a_{j}$ from its initial values. Note that acting on our trigonometric polynomials, the operator $\partial_{\theta}$ is bounded.

These computations are summarized by the quasilinear versions of Theorems 4.1 and 4.2.

Theorem 5.1: If the eikonal, coherence, and strong finiteness hypotheses are satisfied and $u^{\epsilon}$ is given by (4.1) with $p=1 /(J-1)$, then

$$
L\left(u^{\epsilon}, \epsilon \partial\right)\left(u^{\epsilon}\right)+F\left(u^{\epsilon}\right) \sim O \quad \text { in } \quad C^{\infty}(\Omega)
$$

if (5.5) and (5.8), and the infinite sequence of equations (5.13) and (5.14) are satisfied.

Theorem 5.2: Suppose in addition that the domain of dependence hypothesis is satisfied, that $T_{1}>0$ and for $j \geqslant 0$

$$
g_{j}(y, \theta) \in C_{0}^{\infty}\left(\mathbf{R}^{d} \times S^{1}\right), \quad \operatorname{supp} g \subset(\Omega \cap\{t=0\}) \times S^{1} \quad \text { and } \quad \Pi g_{j}=g_{j} .
$$

Then there is a $\left.T \in] 0, T_{1}\right]$ and a unique solution $a_{0} \in C_{(0)}^{\infty}\left((\Omega \cap\{0 \leqslant t \leqslant T\}) \times S^{1}\right)$ of (5.5) and (5.8) such that $\Pi a_{0}(0, y)=g_{0}(y)$. With this $T$, there are unique functions $a_{j} \in C_{(0)}^{\infty}\left((\Omega \cap\{0 \leqslant t \leqslant T\}) \times S^{1}\right)$ satisfying the initial conditions $\Pi a_{j}(0, y)=g_{j}(y)$ and the infinite sequence of equations (5.13) and (5.14).

Example: The instantaneous nonlinear response model yields cubic transport equations along rays moving with the group velocity. The resulting nonlinear ordinary differential equations are explicitly solvable and yield the standard expressions for self-phase modulation in nonlinear optics. The computations resemble those for the Lorentz model in Sec. IV B 3 and are omitted. They can be found in Ref. 27.

\section{STABILITY OF THE APPROXIMATE SOLUTIONS}

In Secs. IV and V, infinitely accurate approximate solutions of nonlinear wave equations were constructed. They are accurate in the limit of wavelength $\epsilon$ tending to zero. The approximate solutions have residuals each of whose derivatives converges to zero more rapidly than any power of $\epsilon$. In this sense they are in fact very accurate. Nevertheless it remains to show that there are solutions of the exact equations which are close to the approximate solutions. This is a stability result which asserts that removing the infinitely small residual does not perturb the solution much. The approximate solutions of Secs. IV and V are thereby shown to be asymptotic to exact solutions.

The results of this section are closely related to and were inspired by the important stability results of O. Gues. ${ }^{17,18}$ They differ in two essential ways. First the underlying equation is an operator in $\epsilon \partial$ which makes the problem a little more sensitive. This potential instability is compensated by the conservation hypothesis at the beginning of Sec. III. If the background operator were not conservative (or more generally dissipative), the errors could be amplified by factors of the form $e^{t / \epsilon}$ which would overwhelm the residuals of order $\epsilon^{\infty}$.

In addition to the conservation hypothesis which is essential, we also assume more regularity of the approximate solutions $u^{\epsilon}$ than does Gues. Roughly where he assumes $L^{2}$ bounds on the derivatives $\epsilon \partial$ we assume $L^{\infty}$ bounds. The reason for our choice is that in practice one usually controls very well the approximate solutions so that one has such sup norm bounds, for example in the constructions of Secs. IV and V. Second, this allows a simplification of the proof as was remarked in Ref. 27. Theorems with $L^{2}$ hypotheses like those of Gues are also valid in the dispersive setting.

Consider the quasilinear operator

$$
L(u, \epsilon \partial) u+F(u)
$$


whose nonlinear terms are of order $J$ near $u=0$ in the sense that the following strengthening of (5.2) is satisfied.

$$
|\alpha| \leqslant J-2 \Rightarrow \partial_{u, \bar{u}}^{\alpha} A_{\mu}(0)=0 \quad \text { and } \quad|\beta| \leqslant J-1 \Rightarrow \partial_{u, \bar{u}}^{\beta} F(0)=0 .
$$

This is equivalent to supposing (5.2) is valid for all phases.

Suppose that the family $u^{\epsilon} \in C_{(0)}^{\infty}\left([0, T] \times \mathbf{R}^{d}\right)$ satisfies

$$
L\left(u^{\epsilon}, \partial\right) u^{\epsilon}+F\left(u^{\epsilon}\right)=r^{\epsilon}
$$

with residuals $r^{\epsilon} \sim 0$ in the sense that they are supported in a compact subset of $[0, T] \times \mathbf{R}^{d}$ independent of $\epsilon$ and for all $\alpha$ and $n$

$$
\lim _{\epsilon \rightarrow 0} \epsilon^{-n}\left\|\partial_{x}^{\alpha} r^{\epsilon}\right\|_{L^{\infty}\left([0, T] \times \mathbf{R}^{d}\right)}=0 .
$$

The goal is to compare $u^{\epsilon}$ to solutions $v^{\epsilon}$ of the initial value problem

$$
L\left(v^{\epsilon}, \partial\right) v^{\epsilon}+F\left(v^{\epsilon}\right)=h^{\epsilon}, \quad v^{\epsilon}(0, y)=u^{\epsilon}(0, y)+g^{\epsilon}(y),
$$

where

$$
h^{\epsilon} \sim 0 \text { in } C_{(0)}^{\infty}\left([0, T] \times \mathbf{R}^{d}\right) \text { and } \quad g^{\epsilon} \sim 0 \text { in } C_{(0)}^{\infty}\left(\mathbf{R}^{d}\right) .
$$

The case of $h^{\epsilon}=0$ and $g^{\epsilon}=0$ is especially interesting but no easier than (6.5). The standard existence theorem for quasilinear hyperbolic equations with smooth coefficients and data implies the existence and unicity of a regular solution $v^{\epsilon}$ on $\left[0, T_{\epsilon}\left[\times \mathbf{R}^{d}\right.\right.$ for a possibly small positive $T_{\epsilon}$. The goal is to prove that this time of existence is greater than or equal to $T$, and that $v^{\epsilon}-u^{\epsilon} \sim 0$ on $[0, T] \times \mathbf{R}^{d}$. Since the $H^{s}$ norms of the data explode as soon as $s>p$, the standard local existence theorems yield a domain of existence which shrinks toward $\{t=0\}$ when $\epsilon \rightarrow 0$.

In Secs. IV and V, approximate solutions were constructed with the form $\epsilon^{p} a(\epsilon, x, \phi(x) / \epsilon)$ where the profile or envelope $a$ is smooth and periodic in $\theta$. Here $p=1 /(J-1)$ is the critical exponent. These are special examples of (6.2) and (6.3). With the above notation the statements for the quasilinear and semilinear cases are identical.

Theorem 6.1: Suppose that $p=1 /(J-1), u^{\epsilon}=\epsilon^{p} U^{\epsilon}$ satisfies (6.2), (6.3) and for all $\alpha$

$$
\sup _{0<\epsilon<1}\left\|\left(\epsilon \partial_{x}\right)^{\alpha} U^{\epsilon}\right\|_{L^{\infty}\left([0, T] \times \mathbf{R}^{d}\right)}<\infty \quad \text { and } \sup _{\substack{0<\epsilon<1 \\ 0 \leqslant t \leqslant T}}\left\|\left(\epsilon \partial_{x}\right)^{\alpha} U^{\epsilon}(t)\right\|_{L^{2}\left(\mathbf{R}^{d}\right)}<\infty \text {. }
$$

Then there is an $\left.\epsilon_{0} \in\right] 0,1\left[\right.$ so that for $\epsilon<\epsilon_{0}$ the solution $v^{\epsilon}$ to (6.4) exists and is smooth on $[0, T] \times \mathbf{R}^{d}$ and in addition

$$
v^{\epsilon} \sim u^{\epsilon} \text { in } C_{(0)}^{\infty}\left([0, T] \times \mathbf{R}^{d}\right) .
$$

Note that the family of approximate solutions $\left\{u^{€}\right\}$ is not bounded in $H^{s}$ as soon as $s>p$. The fact that the approximate solutions are large in these norms is the source of the difficulty.

Proof: The proof in the semilinear case, that is when the coefficients of $L$ do not depend on $u$, contains the main ideas and is somewhat simpler. For that reason we present first the proof in the semilinear case, and then present the quasilinear case.

Step 1. Taylor expansion absorbs the critical exponent: Define $V^{\epsilon}, W^{\epsilon}$, and $w^{\epsilon}$ by

$$
v^{\epsilon}=\epsilon^{p} V^{\epsilon}, \quad w^{\epsilon}=v^{\epsilon}-u^{\epsilon}, \quad \text { and } \quad W^{\epsilon}=V^{\epsilon}-U^{\epsilon} .
$$


The large letter is $\epsilon^{p}$ times the small. The equation for $v^{\epsilon}$ is equivalent to the following initial value problem for $W^{\epsilon}$,

$$
L(\epsilon \partial) \epsilon^{p} W^{\epsilon}+F\left(\epsilon^{p} U^{\epsilon}+\epsilon^{p} W^{\epsilon}\right)-F\left(\epsilon^{p} U^{\epsilon}\right)=h^{\epsilon}-r^{\epsilon}, \quad W^{\epsilon}(0, y)=\epsilon^{-p} g \epsilon .
$$

The right-hand sides are $\sim 0$.

Write

$$
F(u+w)-F(u)=G(u, w) w, \quad G(u, w) w:=\int_{0}^{1} F_{u}(u+s w) d s .
$$

Then $G(u, w)$ is a smooth matrix valued function whose derivatives of order $\leqslant J-2$ vanish at the origin. Taylor's theorem yields

$$
G(\zeta)=\int_{0}^{1} \frac{(1-s)^{j-2}}{(j-2) !}\left(\frac{d}{d s}\right)^{j-1} G(s \zeta) d s=\int_{0}^{1} \frac{(1-s)^{j-2}}{(j-2) !}\left(\left(\zeta \cdot \partial_{\zeta}\right)^{j-1} G\right)(s \zeta) d s
$$

The factors $\zeta$ on the right show that each element of the matrix $G$ is the product of a polynomial homogeneous of degree $J-1$ with a smooth function of $u, w$. Therefore

$$
F\left(\epsilon^{p} U^{\epsilon}+\epsilon^{p} W^{\epsilon}\right)-F\left(\epsilon^{p} U^{\epsilon}\right)=\epsilon^{p J} H\left(\epsilon, U^{\epsilon}, W^{\epsilon}\right) W^{\epsilon},
$$

where $H$ is a smooth matrix-valued function of its arguments.

Plug this into (6.9) and divide by $\epsilon^{p+1}=\epsilon^{p J}$ to find the singular system

$$
\left(L_{1}\left(\partial_{x}\right)+\frac{1}{\epsilon} L_{0}\right) W^{\epsilon}+H\left(\epsilon, U^{\epsilon}, W^{\epsilon}\right) W^{\epsilon}=\frac{h^{\epsilon}-r^{\epsilon}}{\epsilon^{p J}} .
$$

Step 2: $H_{\epsilon}^{s}$ estimates for the singular linear operator. Though the $L_{0}$ term in (6.11) has a coefficient which explodes, the matrix $L_{0}$ is antisymmetric so this term does not lead to explosion of $L^{2}$ norms. Also the division of the term on the right by $\epsilon^{p J}$ is not dangerous since $h^{\epsilon}-r^{\epsilon} \sim 0$. It remains to avoid the difficulties posed by the fact that the derivatives of $U^{\epsilon}$ are large. This prevents one from simply differentiating the equation (6.11). The remedy is to apply derivatives $\epsilon \partial$ and use (6.6).

The first remark in the previous paragraph shows that there is a $C>0$ so that for all $0 \leqslant t, 0<\epsilon$ and all $w \in C_{(0)}^{\infty}\left([0, t] \times \mathbf{R}^{d}\right)$

$$
\|w(t)\|_{L^{2}\left(\mathbf{R}^{d}\right)} \leqslant C\left(\|w(0)\|_{L^{2}\left(\mathbf{R}^{d}\right)}+\int_{0}^{t}\left\|\left(L_{1}(\partial)+\frac{1}{\epsilon} L_{0}\right) w(\sigma)\right\|_{L^{2}\left(R^{d}\right)} d \sigma\right) .
$$

For integer $s \geqslant 0$ define a family of norms each equivalent to the norm in $H^{s}\left(\mathbf{R}^{d}\right)$ by

$$
\|w\|_{H_{\epsilon}^{s}\left(\mathbf{R}^{d}\right)}^{2}:=\sum_{|\alpha| \leqslant s}\left\|\left(\epsilon \partial_{y}\right)^{\alpha} w\right\|_{L^{2}\left(\mathbf{R}^{d}\right)}^{2} .
$$

Commuting with the operators $\epsilon \partial_{y}$ shows that with the same constant as in (6.13) one has

$$
\|w(t)\|_{H_{\epsilon}^{s}\left(\mathbf{R}^{d}\right)} \leqslant C\left(\|w(0)\|_{H_{\epsilon}^{s}\left(\mathbf{R}^{d}\right)}+\int_{0}^{t}\left\|\left(L_{1}(\partial)+\frac{1}{\epsilon} L_{0}\right) w(\sigma)\right\|_{H_{\epsilon}^{s}\left(\mathbf{R}^{d}\right)} d \sigma\right) .
$$

For $s>d / 2$, a straightforward scaling yields the Sobolev estimate, 


$$
\|w\|_{L^{\infty}\left(\mathbf{R}^{d}\right)} \leqslant C(s) \epsilon^{-d / 2}\|w\|_{H_{\epsilon}^{s}\left(\mathbf{R}^{d}\right)} .
$$

Step 3. Estimate for the nonlinear term: The remaining key ingredient in the proof is the following estimate for the nonlinear term.

Lemma 6.2: Suppose that $U^{\epsilon} \in C_{(0)}^{\infty}\left([0, T] \times \mathbf{R}^{d}\right)$ satisfies (6.6). Then there is a constant $C=C\left(s, U^{\epsilon}\right)$ so that for all $\left.\left.\sigma \in[0, T], \epsilon \in\right] 0,1\right]$, and $W \in H^{s}\left(\mathbf{R}^{d}\right)$ satisfying

$$
\|W\|_{L^{\infty}\left(\mathbf{R}^{d}\right)} \leqslant 1,
$$

one has

$$
\left\|H\left(\epsilon, U^{\epsilon}, W\right) W\right\|_{H_{\epsilon}^{s}\left(\mathbf{R}^{d}\right)} \leqslant C\|W\|_{H_{\epsilon}^{s}\left(\mathbf{R}^{d}\right)} .
$$

Proof of Lemma: With $|\alpha| \leqslant s$, Leibniz's rule shows that $\left(\epsilon \partial_{y}\right)^{\alpha}\left\{H\left(\epsilon, U^{\epsilon}, W\right) W\right\}$ is a finite sum of terms of the form

$$
H^{\delta}\left(\epsilon, U^{\epsilon}, W\right) \prod_{j}\left(\epsilon \partial_{y}\right)^{\beta_{j}} U^{\epsilon} \prod_{k}\left(\epsilon \partial_{y}\right)^{\gamma_{k} W}, \quad \sum \beta_{j}+\sum \gamma_{k}=\alpha,
$$

where there is always at least one $\gamma_{k}$. Assumptions (6.6) and (6.17) guarantee that all the factors except the derivatives of $w$ are bounded. To estimate the product of the derivatives of $W$, use the following result.

Gagliardo-Nirenberg $\epsilon$-inequalities: If $W \in H^{r}\left(\mathbf{R}^{d}\right) \cap L^{\infty}\left(\mathbf{R}^{d}\right)$ and $0<|\alpha|<r$, then

$$
\partial_{y}^{\alpha} W \in L^{2 r /|\alpha|}\left(\mathbf{R}^{d}\right)
$$

Moreover, there is a constant $C=C(|\alpha|, s, d)$ so that for all $\epsilon \in] 0, \infty[$,

$$
\left\|\left(\epsilon \partial_{y}\right)^{\alpha} W\right\|_{L^{2 r|| \alpha \mid}\left(\mathbf{R}^{d}\right)} \leqslant C\|W\|_{L^{\infty}\left(\mathbf{R}^{d}\right)}^{1-|\alpha| \mid r}\left(\sum_{|\beta|=r}\left\|\left(\epsilon \partial_{y}\right)^{\beta} W\right\|_{L^{2}\left(\mathbf{R}^{d}\right)}\right)^{|\alpha| / r} .
$$

Proof of Gagliardo-Nirenberg $\epsilon$-inequality: The inequality is classical for $\epsilon=1$. Applying that inequality to $V(x):=W(\epsilon x)$ proves (6.20).

Let $r:=\Sigma\left|\gamma_{k}\right| \leqslant s$. The Gagliardo-Nirenberg inequalities imply that

$$
\left\|\left(\epsilon \partial_{y}\right){ }^{\gamma_{k}} W\right\|_{L^{2 r|| \gamma_{k} \mid} \leqslant C\|W\|_{H_{\epsilon}^{s}\left(\mathbf{R}^{d}\right)}^{\left|\gamma_{k}\right| / r}} .
$$

Hölder's inequality shows that

$$
\left\|\prod_{k}\left(\epsilon \partial_{y}\right)^{\gamma_{k} W}\right\|_{L^{2}\left(\mathbf{R}^{d}\right)} \leqslant C\|W\|_{H_{\epsilon}^{s}\left(\mathbf{R}^{d}\right)},
$$

and the proof of the Lemma is complete.

Step 4. End of semilinear proof: We proceed to estimate $W^{\epsilon}$ for $0 \leqslant t \leqslant T_{*}(\epsilon) \leqslant T$ where

$$
T_{*}(\epsilon):=\sup \left\{t \in[0, T]:\left\|W^{\epsilon}\right\|_{L^{\infty}\left([0, t] \times \mathbf{R}^{d}\right)} \leqslant 1\right\} .
$$

Since $W^{\epsilon}(0,.) \sim 0$ in $C_{0}^{\infty}\left(\mathbf{R}^{d}\right)$ one can choose $\epsilon_{1}>0$ so that

$$
\epsilon<\epsilon_{1} \Rightarrow\left\|W^{\epsilon}(0)\right\|_{L^{\infty}\left(\mathbf{R}^{d}\right)}<1 .
$$

Then $T_{*}(\epsilon)>0$ for $\epsilon<\epsilon_{1}$.

For $0 \leqslant t \leqslant T_{*}$ the basic energy inequality implies that 


$$
\left\|W^{\epsilon}(t)\right\|_{H_{\epsilon}^{s}} \leqslant C\left(\left\|W^{\epsilon}(0)\right\|_{H_{\epsilon}^{s}}+\int_{0}^{t}\left\|H\left(\epsilon, U^{\epsilon}(t), W^{\epsilon}(t)\right) W^{\epsilon}(t)-\frac{h^{\epsilon}(t)-r^{\epsilon}(t)}{\epsilon^{p}}\right\|_{H_{\epsilon}^{s}} d t\right) .
$$

Using the Lemma and (6.5) yields for all $n$

$$
\left\|W^{\epsilon}(t)\right\|_{H_{\epsilon}^{s} \leqslant C(s, n)}\left(\epsilon^{n}+\int_{0}^{t}\left(\left\|W^{\epsilon}(t)\right\|_{H_{\epsilon}^{s}}+\epsilon^{n}\right) d t\right) .
$$

Gronwall's inequality implies

$$
\left\|W^{\epsilon}(t)\right\|_{H_{\epsilon}^{s} \leqslant C(s, n, T) \epsilon^{n}}
$$

First take $s=n>d / 2$ and choose $\epsilon_{0} \leqslant \epsilon_{1}$ so that (6.22) in concert with Sobolev's inequality (6.16) implies that $\left\|W^{\epsilon}(t)\right\|_{L^{\infty}\left(\mathbf{R}^{d}\right)} \leqslant \frac{1}{2}$. It then follows that for $\epsilon \leqslant \epsilon_{0}, T_{*}(\epsilon)=T$ which proves that $v^{\epsilon}$ exists for $0 \leqslant t \leqslant T$ and that inequality (6.22) holds throughout this region.

This done, choose $s>d / 2+|\alpha|$ Then Sobolev's inequality implies

$$
\begin{aligned}
\left\|\partial_{y}^{\alpha} W^{\epsilon}(t)\right\|_{L^{\infty}\left(\mathbf{R}^{d}\right)} & \leqslant C(s) \epsilon^{-d / 2}\left\|\partial_{y}^{\alpha} W^{\epsilon}(t)\right\|_{H_{\epsilon}^{s}\left(\mathbf{R}^{d}\right)} \\
& \leqslant C(s) \epsilon^{-|\alpha|-d / 2}\left\|W^{\epsilon}\right\|_{H_{\epsilon}^{s+|\alpha|}\left(\mathbf{R}^{d}\right)} \leqslant C(s, n, T) \epsilon^{n-d / 2-|\alpha|} .
\end{aligned}
$$

Since this is true for all $n$, this shows that all the $y$ derivatives of $W^{\epsilon}$ are $O\left(\epsilon^{\infty}\right)$.

To obtain the same result for the time derivatives of $W^{\epsilon}$ it suffices to use the differential equation to express these derivatives in terms of $y$ derivatives. This shows that all derivatives of $W^{\epsilon}$ are $O\left(\epsilon^{\infty}\right)$ in sup norm, and therefore the proof in the semilinear case is complete.

Step 5. Taylor's theorem and the quasilinear terms: In the quasilinear case, the initial value problem for $v^{\epsilon}$ is equivalent to the following initial value problem for $W^{\epsilon}$ :

$$
\begin{gathered}
L\left(\epsilon^{p} U^{\epsilon}+\epsilon^{p} W^{\epsilon}, \epsilon \partial\right) \epsilon^{p} W^{\epsilon}+F\left(\epsilon^{p} U^{\epsilon}+\epsilon^{p} W^{\epsilon}\right)-F\left(\epsilon^{p} U^{\epsilon}\right)+\left(L\left(\epsilon^{p} U^{\epsilon}+\epsilon^{p} W^{\epsilon}, \epsilon \partial\right)\right. \\
\left.-L\left(\epsilon^{p} U^{\epsilon}, \epsilon \partial\right)\right) \epsilon^{p} U^{\epsilon}=h^{\epsilon}-r^{\epsilon}, \\
W^{\epsilon}(0, y)=\epsilon^{-p} g{ }^{\epsilon} \sim 0 .
\end{gathered}
$$

The coefficients in the last difference on the left of (6.23) are of the form

$$
A_{\mu}\left(\epsilon^{p} U^{\epsilon}+\epsilon^{p} W^{\epsilon}\right)-A_{\mu}\left(\epsilon^{p} U^{\epsilon}\right)
$$

where $A_{\mu}$ vanishes to order $J-1$ at the origin. The Taylor theorem argument of step 1 shows this difference is of the form

$$
\epsilon^{p(J-1)} H_{\mu}\left(\epsilon, U^{\epsilon}, W^{\epsilon}\right) W^{\epsilon}
$$

Thus the last difference on the right has the form

$$
\sum_{\mu} \epsilon^{p(J-1)} H_{\mu}\left(\epsilon, U^{\epsilon}, W^{\epsilon}\right) W^{\epsilon} \epsilon \partial_{\mu} \epsilon^{p} U^{\epsilon}:=\epsilon^{p J} H\left(\epsilon, U^{\epsilon}, \epsilon \partial U^{\epsilon}, W^{\epsilon}\right) W^{\epsilon}
$$

This yields the equation

$$
\left(L_{1}\left(v^{\epsilon}, \partial\right)+\frac{1}{\epsilon} L_{0}\right) W^{\epsilon}+H\left(\epsilon, U^{\epsilon}, \epsilon \partial U^{\epsilon}, W^{\epsilon}\right) W^{\epsilon} \sim 0 .
$$


Step 6. $H_{\epsilon}^{s}$ estimates in the quasilinear case: This is the part of the quasilinear argument which really requires more work than the semilinear case. The problem is that the linear operator

$$
\mathscr{C}(\epsilon, x, \partial):=L_{1}\left(v^{\epsilon}(x), \partial\right)+\frac{1}{\epsilon} L_{0}
$$

in (6.25) has variable coefficients. $\mathscr{C}_{\mu}(\epsilon, x):=A_{\mu}\left(v^{\epsilon}(x)\right)$.

The first step is to remark that it is the Lipshitz norm of the coefficients which is important. This is a consequence of the classical energy identity

$$
\partial_{t} \int_{\mathbf{R}^{d}}\left\langle u(t), A_{0} u(t)\right\rangle d y=2 \operatorname{Re} \int_{\mathbf{R}}^{d}\langle u(t), \mathscr{L} u(t)\rangle d y+\int_{\mathbf{R}^{d}}\left\langle u(t),\left(\sum \frac{\partial \mathscr{C}_{\mu}}{\partial x_{\mu}}\right) u(t)\right\rangle d y .
$$

The coefficients of $\mathscr{L}$ are of the form $A\left(\epsilon^{p} V^{\epsilon}\right)$. Hypothesis (6.1) shows that this coefficient is of the form

$$
A(0)+\epsilon^{p(J-1)} H\left(\epsilon, V^{\epsilon}\right)
$$

Recall that $p(J-1)=1$, so the derivates of such an expression are sums of terms of the form

$$
(\partial H)\left(\epsilon, V^{\epsilon}\right) \epsilon \partial V^{\epsilon}
$$

Therefore, the sup norm of the derivatives of the coefficients is bounded by the sup norm of $V^{\epsilon}$ and the $\epsilon \partial$ derivative of $V^{\epsilon}$, that is

$$
\left\|\left(\sum_{\mu=0}^{d} \frac{\partial \mathscr{C}_{\mu}}{\partial x_{\mu}}\right)\right\|_{L^{\infty}\left([0, T] \times \mathbf{R}^{d}\right)} \leqslant C\left(\left\|V^{\epsilon}\right\|_{L^{\infty}\left([0, T] \times \mathbf{R}^{d}\right)}+\sum_{\mu}\left\|\epsilon \partial_{\mu} V^{\epsilon}\right\|_{L^{\infty}\left([0, T] \times \mathbf{R}^{d}\right)}\right) .
$$

This suggests the introduction of the following definition.

Definition: $O n$

$$
\Omega_{T}:=[0, T] \times \mathbf{R}^{d},
$$

the family of norms, each equivalent to the Lipshitz norm, is defined by

$$
\|V\|_{\operatorname{Lip}_{\epsilon}\left(\Omega_{T}\right)}:=\|V\|_{L^{\infty}\left(\Omega_{T}\right)}+\sup _{0 \leqslant \mu \leqslant d}\left\|\epsilon \partial_{\mu} V\right\|_{L^{\infty}\left(\Omega_{T}\right)} .
$$

The preceding computations prove the following basic $L^{2}$ estimate.

Proposition 6.3: For any $K, T>0$ so that $L$ is symmetric hyperbolic on $|u| \leqslant K$, there is a constant $C=C(K, L)$ so that if $T>0$ and $v^{\epsilon}=\epsilon^{p} V^{\epsilon} \in C_{(0)}^{\infty}\left(\Omega_{T}\right)$ satisfies

$$
\left\|V^{\epsilon}\right\|_{\operatorname{Lip}_{\epsilon}\left(\Omega_{T}\right)} \leqslant K
$$

then for all $0 \leqslant t \leqslant T, 0<\epsilon<\infty$ and $W \in C_{(0)}^{\infty}\left(\Omega_{t}\right)$,

$$
\|W(t)\|_{L^{2}\left(\mathbf{R}^{d}\right)} \leqslant C\left(\|W(0)\|_{L^{2}\left(\mathbf{R}^{d}\right)}+\int_{0}^{t}\left\|\left(L_{1}\left(v^{\epsilon}, \partial\right)+\frac{1}{\epsilon} L_{0}\right) W(\sigma)\right\|_{L^{2}\left(\mathbf{R}^{d}\right)} d \sigma\right) .
$$

This estimate is used for $W:=(\epsilon \partial)^{\alpha} W^{\epsilon}$ in which case the right-hand side is estimated as follows. Define 


$$
T_{*}(\epsilon):=\sup \left\{t \in[0, T]:\left\|W^{\epsilon}\right\|_{\operatorname{Lip}_{\epsilon}\left(\Omega_{t}\right)} \leqslant 1\right\} .
$$

Lemma 6.4: With the shorthand $Z:=\epsilon \partial_{y}$, if $|\alpha| \leqslant s \in \mathbf{N}$, there is a constant $C=C\left(s, U^{\epsilon}\right)$ so that if $0<t \leqslant T_{*}(\epsilon)$, then

$$
\left\|\left(L_{1}\left(v^{\epsilon}, \partial\right)+\frac{1}{\epsilon} L_{0}\right) Z^{\alpha} W^{\epsilon}(t)\right\|_{H_{\epsilon}^{s}\left(\mathbf{R}^{d}\right)} \leqslant C\left\|W^{\epsilon}(t)\right\|_{H_{\epsilon}^{s}\left(\mathbf{R}^{d}\right)}+O\left(\epsilon^{\infty}\right) .
$$

Proof of Lemma 6.4: Apply $Z^{\alpha}$ to (6.25). Expand the $Z^{\alpha} H$ term using Leibniz's rule and then use the $\epsilon$-Gagliardo-Nirenberg estimates as in the proof of Lemma 6.2 to show that

$$
\left\|Z^{\alpha}\left\{H\left(\epsilon, U^{\epsilon}, \epsilon \partial U^{\epsilon}, W^{\epsilon}\right) W^{\epsilon}\right\}\right\|_{L^{2}\left(\mathbf{R}^{d}\right)} \leqslant C\left\|W^{\epsilon}(t)\right\|_{H_{\epsilon}^{s}\left(\mathbf{R}^{d}\right)} .
$$

To prove Lemma 6.4 it suffices to prove the following commutator estimate:

$$
\left\|\left[L_{1}\left(v^{\epsilon}, \partial\right)+\frac{1}{\epsilon} L_{0}, Z^{\alpha}\right] W^{\epsilon}\right\|_{L^{2}\left(\mathbf{R}^{d}\right)} \leqslant C\left\|W^{\epsilon}\right\|_{H_{\epsilon}^{s}\left(\mathbf{R}^{d}\right)} .
$$

The commutators with $L_{0}$ and with $A_{\mu}(0) \partial_{\mu}$ vanish identically. Using (6.27) and $p(J-1)=1$ shows that what remains to be estimated is $\left[Z^{\alpha}, H\left(\epsilon, U^{\epsilon}+W^{\epsilon}\right) \epsilon \partial_{\mu}\right] W^{\epsilon}$. Leibniz's rule shows that this is equal to a sum of terms of the form

$$
G\left(\epsilon, U^{\epsilon}, V^{\epsilon}\right)\left(\prod_{j} Z^{\alpha_{j}} U^{\epsilon}\right)\left(\prod_{k} Z^{\beta_{k}} W^{\epsilon}\right) Z^{\gamma} \epsilon \partial_{\mu} W^{\epsilon}, \quad \gamma+\sum \alpha_{j}+\sum \beta_{k}=\alpha, \quad|\gamma|<s .
$$

Note that there are $s+1$ derivatives in total. The $G$ term and the product of the derivatives of $U^{\epsilon}$ each belongs to $L^{\infty}$ so it suffices to estimate the $L^{2}$ norm of the remaining factors.

First consider the case $\mu \neq 0$. If $\left|\beta_{k}\right| \leqslant 1$ for all $k$, estimate the $L^{2}$ norm of $Z^{\gamma} \epsilon \partial_{\mu} W^{\epsilon}$ by the $H_{\epsilon}^{s}$ norm of $W^{\epsilon}$ and the rest of the factors in $L^{\infty}$. Otherwise, include the $Z^{\gamma} \epsilon \partial_{\mu}$ term as one of the $\beta$ terms. It suffices to show that

$$
\sum \beta_{k} \leqslant s+1, \quad\left|\beta_{k}\right| \leqslant s \Rightarrow\left\|\left(\prod_{\left|\beta_{k}\right| \geqslant 2} Z^{\beta_{k}} W^{\epsilon}\right)\right\|_{L^{2}\left(\mathbf{R}^{d}\right)} \leqslant C\left\|W^{\epsilon}\right\|_{H_{\epsilon}^{s}\left(\mathbf{R}^{d}\right)} .
$$

Define

$$
r:=1+\sum_{\left|\beta_{k}\right| \geqslant 2}\left(\left|\beta_{k}\right|-1\right) \leqslant s .
$$

Each factor is a $Z$ derivative of order $|\beta|-1$ of a $Z W^{\epsilon} \in H_{\epsilon}^{r-1}$. The Gagliardo-Nirenberg inequality applied to $W:=Z W^{\epsilon}$ yields

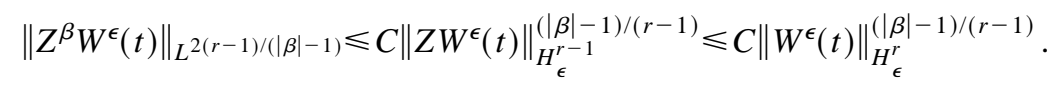

Hölder's inequality yields

$$
\left\|\left(\prod_{\left|\beta_{k}\right| \geqslant 2} Z^{\beta_{k} W^{\epsilon}}\right)\right\|_{L^{2}\left(\mathbf{R}^{d}\right)} \leqslant C\left\|W^{\epsilon}(t)\right\|_{H_{\epsilon}^{r}\left(\mathbf{R}^{d}\right)} \leqslant C\left\|W^{\epsilon}(t)\right\|_{H_{\epsilon}^{s}\left(\mathbf{R}^{d}\right)} .
$$


Finally, if $\mu=0$ in (6.34) use Eq. (6.25) to express the $\epsilon \partial_{t}$ derivative of $W^{\epsilon}$ in terms of $\epsilon \partial_{j}$ derivatives plus a term $\epsilon H\left(\epsilon, U^{\epsilon}, \epsilon \partial U^{\epsilon}, W^{\epsilon}\right) W^{\epsilon}$ plus a $O\left(\epsilon^{\infty}\right)$. Each of the terms produced can be analyzed by the methods above so the proof of Lemma 6.4 is complete.

End of quasilinear proof: Applying (6.29) and (6.34) together with Gronwall's inequality shows that for all positive integers $n$ and $s$ there is a constant $C(n, s)$ so that for all $0 \leqslant t \leqslant T_{*}$,

$$
\sum_{|\alpha| \leqslant s}\left\|\left(\epsilon \partial_{x}\right)^{\alpha} W^{\epsilon}(t)\right\|_{L^{2}\left(\mathbf{R}^{d}\right)} \leqslant C \epsilon^{n} .
$$

Thanks to (6.24) and (6.25) for the time derivative, one can choose $\epsilon_{1}>0$ so that for $\epsilon \leqslant \epsilon_{1}$

$$
\sum_{|\alpha| \leqslant 1}\left\|Z^{\alpha} W^{\epsilon}(0)\right\|_{L^{\infty}\left(\mathbf{R}^{d}\right)}<\frac{1}{2}
$$

Then $T_{*}(\epsilon)>0$ for $\epsilon<\epsilon_{1}$.

Take $s=n>1+d / 2$ and choose $\epsilon_{0} \leqslant \epsilon_{1}$ so that (6.37) in concert with (6.25) and Sobolev's inequality (6.16) implies that

$$
\left\|W^{\epsilon}(t)\right\|_{\operatorname{Lip}_{\epsilon}\left(\Omega_{T_{*}(\epsilon)}\right)} \leqslant \frac{1}{2}
$$

It then follows that for $\epsilon \leqslant \epsilon_{0}, T_{*}(\epsilon)=T$ which proves that $v^{\epsilon}$ exists for $0 \leqslant t \leqslant T$ and that inequality (6.37) holds throughout this region. As at the end of the semilinear case, this implies the conclusion (6.7) and the proof of Theorem 6.1 is complete.

\section{ACKNOWLEDGMENTS}

We have been very fortunate to have had help from a variety of different sources. P. A. Raviart suggested that we study problems from nonlinear optics with the methods of nonlinear asymptotics. His continuing support has buoyed our efforts throughout. Many members of the Groupe de Recherche POAN (Propagation d'Ondes dans les Milieux Aléatoirees et/ou Non Linéaires) and the Center for Ultrafast Optics in Ann Arbor have taught us innumerable things and we would like to especially thank A. Migus, G. Mourou, C. Gouédard, X. Liu, and A. Braun, who have helped us try to bridge the gap between physics and mathematics. Our mathematical colleagues, especially G. Metivier, J. L. Joly, M. Weinstein, G. Papanicolaou, J. Hunter, O. Gues, J. C. Saut, have generously offered their advice on many occasions. We have had grant support from the U.S. National Science Foundation and the U.S. Office of Naval Research under Grants No. NSF-DMS-9203413 and No. OD-G-N-0014-92-J-1245, respectively, and also from the NSFCNRS cooperative research Grant No. NSF-INT-9314095. The paper was written while J. Rauch was visiting the École Normale Supérieure in Paris and we would like to thank both the ENS and the CNRS for their support during that period.

\footnotetext{
${ }^{1}$ R. P. Feynmann, R. Leighton, and M. Sands, The Feynmann Lectures on Physics Vols. I, II (Addison-Wesley, Reading, MA, 1964).

${ }^{2}$ L. D. Landau and E. M. Lifshitz, Electrodynamics of Continuous Media, Course of Theoretical Physics, Vol. 8, translated by J. B. Sykes and J. S. Bell (Pergamon, Oxford, 1960).

${ }^{3}$ P. Donnat and J. Rauch, Modelling the dispersion of light, in Singularities and Oscillations, edited by J. Rauch and M. Taylor, IMA Volumes in Mathematics and its Applications, Vol. 91 (Springer-Verlag, New York, 1997).

${ }^{4}$ G. Whitham, Linear and Nonlinear Waves (Wiley, New York, 1974).

${ }^{5}$ Y. Choquet-Bruhat, "Ondes asymptotiques et approchées pour les systèmes d'équations aux dérivées partielles non linéaires," J. Math. Pure Appl. 48, 117-158 (1969).

${ }^{6}$ A. Majda and R. Rosales, "Resonantly interacting weakly nonlinear hyperbolic waves I: a single space variable," Stud. Appl. Math. 71, 149-179 (1984).

${ }^{7}$ J. Hunter and J. Keller, "Weakly nonlinear high frequency waves," Comm. Pure Appl. Math. 36, 547-569 (1983).

${ }^{8}$ J. Hunter, A. Majda, and R. Rosales, "Resonantly interacting weakly nonlinear hyperbolic waves II: several space variables." Stud. Appl. Math. 75, 187-226 (1986).
} 
${ }^{9}$ J. L. Joly and J. Rauch, “Justification of multidimensional single phase semilinear geometric optics,'” Trans. Am. Math. Soc. 330, 599-625 (1992).

${ }^{10}$ J.-L. Joly, G. Metivier, and J. Rauch, "Resonant one dimensional nonlinear geometric optics," J. Funct. Anal. 114, 106-231 (1993).

${ }^{11}$ J.-L. Joly, G. Metivier, and J. Rauch, "Coherent and focusing multidimensional nonlinear geometric optics," Ann. L'École Norm. Supér. Paris 28, 51-113 (1995).

${ }^{12}$ J.-L. Joly, G. Metivier, and J. Rauch, "Generic rigorous asymptotic expansions for weakly nonlinear multidimensional oscillatory waves" Duke Math. J. 70, 373-404 (1993).

${ }^{13}$ J.-L. Joly, G. Metivier, and J. Rauch, "Coherent nonlinear waves and the Wiener algebra," Ann. Inst. Fourier 44, 167-196 (1994).

${ }^{14}$ J.-L. Joly, G. Metivier, and J. Rauch, "Several recent results in nonlinear geometric optics," in Partial Differential Equations and Mathematical Physics, edited by L. Hörmander and A. Melin (Birkhäuser, Basel, 1996).

${ }^{15}$ R. Lewis, “Asymptotic theory of wave propagation," Arch. Rat. Mech. Anal. 20, 192-250 (1965); 25, 406 (1967).

${ }^{16}$ G.-Q. Chen, C. D. Levermore, and T.-P. Liu, "Hyperbolic conservation laws with stiff relaxation terms and entropy, Comm. Pure Appl. Math. XLVII, 787-830 (1994).

${ }^{17}$ O. Gues, "Développements asymptotiques de solutions exactes de systèmes hyperboliques quasilinéaires," Asymptotic Anal. 6, 241-269 (1993).

${ }^{18}$ O. Gues, "Ondes multidimensionnelles $\epsilon$-stratifiées et oscillations," Duke Math. J. 68, 401-446 (1992).

${ }^{19}$ P. Lax, "Asymptotic solutions of oscillatory initial value problems," Duke Math. J. 24, 627-645 (1957).

${ }^{20}$ H. A. Lorentz, Theory of Electrons (Teubner, New York, 1909), reprinted (Dover, Englewood Cliffs, NJ, 1952).

${ }^{21}$ E. Purcell, Electricity and Magnetism (McGraw-Hill, New York, 1965).

${ }^{22}$ R. Boyd, Nonlinear Optics (Academic, San Diego, 1992).

${ }^{23}$ A Owyoung, "The origins of the nonlinear refractive indices of liquids and gases," Ph.D. dissertation, California Institute of Technology, 1971.

${ }^{24}$ L. Hörmander, The Analysis of Linear Partial Differential Operators (Springer-Verlag, New York, 1991).

${ }^{25}$ J. Rauch, Lectures on geometric optics, in IAS Park City Summer Math Institute, 1995 (to appear).

${ }^{26}$ R. Courant, Methods of Mathematical Physics, Vol. II (Interscience, New York, 1963).

${ }^{27}$ P. Donnat, "Quelques contributions mathématiques en optique non linéaire," Ph.D. dissertation, École Polytechnique, Paris, 1994. 